Preprints of the

Max Planck Institute for

Research on Collective Goods

Bonn 2013/18

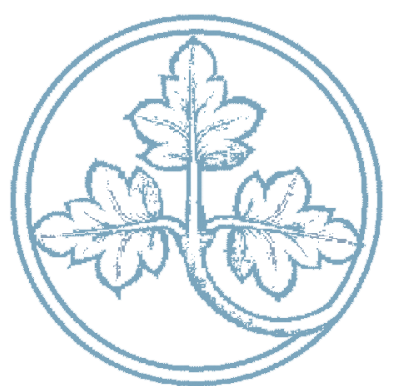

Information Aggregation

Through Stock Prices and the Cost of Capital

Olga Gorelkina

Wolfgang Kuhle

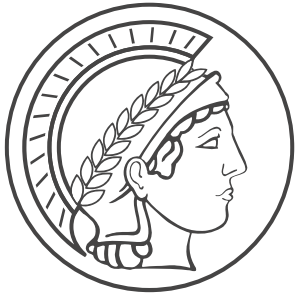




\section{Information Aggregation Through Stock Prices and the Cost of Capital}

Olga Gorelkina / Wolfgang Kuhle

October 2013 


\title{
Information Aggregation Through Stock Prices and the Cost of Capital ${ }^{*}$
}

\author{
Olga Gorelkina, Wolfgang Kuhle ${ }^{\dagger}$
}

October 1, 2013

\begin{abstract}
This paper studies a firm's optimal capital structure in an environment, where the firm's stock price serves as a public signal for its credit worthiness. In equilibrium, equity investors choose how much information to acquire privately, which induces a positive relation between the amount of equity issued and the stock price signal's precision. Thus, through its capital structure, the firm can internalize the informational externality that stock prices exert on bond yields. Firms with a strong fundamental therefore issue more equity and less debt than they would if the informational spill-over did not exist.
\end{abstract}

Keywords: Information Aggregation, Capital Structure, Sequential Markets, Market Depth.

JEL:

C73 D83 G10 G32

\section{Introduction}

Lehman's 2008 bankruptcy may have come as a surprise to those bondholders who believed in its A-ratings. It was less of a surprise to the bondholder who observed that Lehman's stock price had fallen from 62.19 at the beginning of 2008 to its 3.65 low on September 12, 2008, the

${ }^{*}$ We thank Martin Hellwig for his suggestion to think about information spill-overs between stock prices and bond yields. We thank Alia Gizatulina, Dominik Grafenhofer, Kristoffel Grechenig, George Lukyanov, and Martin Szydlowski for comments which improved this paper. The paper also benefited from questions we received in Bonn, CORE Louvain-la-Neuve, and at the PET 2012 and EEA-ESEM 2013 conferences.

${ }^{\dagger}$ Max Planck Institute for Research on Collective Goods, Kurt-Schumacher-Str. 1053113 Bonn, Germany. 
day before Lehman's bankruptcy. Similarly, AIG's A-ratings were not more informative during the days before its bail-out. Again, the stock price, which had fallen by more than 90 percent in that year, provided a more informative signal. In both cases, the stock price served as a timely, costless, and arguably unbiased monitoring device for bondholders.

The present paper studies the informational externality that stock prices exert on equilibrium bond yields. Since stock price and bond yield are both related to a firm's cost of capital, we assume the perspective of a firm which chooses its capital structure to minimize its expected cost of capital. In the present model, this firm will have an incentive to internalize the informational externality that stock prices have on equilibrium bond yields through an optimal choice of its capital structure. The main finding indicates that the informational spill-over from stock price signals to equilibrium bond yields makes it optimal for firms which are financially strong to issue more equity and less debt than they would in a world without the information spill-over. This finding relies on a positive relation between the informativeness of a firm's stock price and the amount of equity issued: as the firm issues more equity, it incentivises equity investors to acquire more private information on the firm's financial health. As a consequence, the firm's stock price becomes more informative and communicates the true financial health of the firm more clearly to Bayesian bond investors. In turn, these bond investors use the information contained in the stock price to calculate the firm's default risk, and the corresponding equilibrium bond yield. Compared to capital structure models ${ }^{1}$ where the management's choice of an optimal capital structure communicates insider knowledge to outside investors, the present paper analyzes how different capital structures facilitate/optimize the information exchange between outside investors.

More precisely, we study a framework where a firm issues bonds $B$ and sells a number of shares $K$ to raise a given target revenue $I$. The firm's objective is to minimize the capital cost $C=K(\theta-p)+R B$ subject to the revenue requirement. The pay-out to equity investors $\theta$ represents the firms financial strength. The model is sequential and at the beginning of time, the firm announces a capital structure $(K, B)$. Subsequently, the stock market opens and the shares $K$ are sold at a market-clearing price $p$ to risk-averse investors who possess private information on the firm's health $\theta$. This stock price aggregates the stock investor's dispersed

\footnotetext{
${ }^{1}$ See Harris and Raviv (1991), pp. 306-315, for a survey of models where various aspects of asymmetric information shape optimal capital structures.
} 
private knowledge and partially reveals the firm's health $\theta$. In turn, the bond market opens and risk averse investors, who observe the price signal $p$, buy the firm's bonds at a market-clearing net interest rate $R(p)$. Bondholders and investors receive their respective pay-outs in the final period when the true strength $\theta$ of the firm is revealed. Bonds pay the net return $R$ if the firms' financial strength is sufficient. Otherwise the firm declares bankruptcy, in which case bond investors incur a loss $L$ on each bond.

The predictions of our model will rely on two main components. First, we assume that the stock market is "more liquid than the bond market", in the sense that there always exists a recent stock price which serves as a noisy signal for bond investors when the bond market opens.2 In our two-period model, this is captured by the assumption that the stock market opens earlier than the bond market. The second main element of our analysis concerns the information acquisition of the Bayesian stock investors who buy shares to maximize expected utility. At a cost, these investors can improve the precision of the information which they learn. As the firm issues more equity $K$, the equity investors' equilibrium net position in the firm increases and investors find it optimal to acquire more information. As a consequence, the price equilibrium, which publicly reveals the information collected by private investors through the market clearing price $p$, becomes more informative. That is, as the firm issues more equity $K$, which reduces its indebtedness $B$, the market price $p$ communicates the firm's fundamental $\theta$ more clearly. In turn, the expected equilibrium bond yield will be lower (higher) if the firm's fundamental is strong (weak) and firms will have an incentive to issue more (less) equity than they would without the informational externality.

Compared to the literature, our analysis of the stock market builds on the Grossman and Stiglitz (1976, 1980), Green (1975), and Hellwig (1980) models of imperfect information aggregation in competitive markets. Unlike these studies, however, the present model does not rely on noise traders to prevent prices from being perfectly revealing. Instead, we present a specification where uncoordinated information acquisition by investors induces an endogenous correlation of the agents' signals. Due to this correlation, prices reveal the true strength of the firm $\theta$ only partially. One assumption in our baseline setting is that equity investors cannot condition their demands on the informational content of prices. In Section 4.4 , we show that

\footnotetext{
${ }^{2}$ In support of this assumption, we note that the financial data provided by Google Finance, Reuters, or Bloomberg make it straightforward to receive stock price information, but difficult to inquire bond yields. That is, stock prices seem to be the main source of aggregate information.
} 
our results also obtain under the alternative assumption which allows for such conditioning. ${ }^{3}$

In turn, this price signal influences the equilibrium rate of return on the firm's debt. This spill-over, which is the main contribution of the paper, influences the firm's optimal capital structure and induces healthy firms to issue more equity than they would if the informational spill-over did not exist. The characterization of this spill-over provides a new aspect to the literature on optimal capital structures in models with default. That is, we add informational spill-overs from stock prices to bond yields to aspects such as agency costs, corporate control considerations, or the tax-shield-default tradeoff. $]^{4}$ In terms of the survey on capital structures by Harris and Raviv (1991), the present analysis is closest to models of asymmetric information, where the management's choice of a capital structure transmits insider information to outside investors. However, instead of a transfer of information from insiders to outsiders, the present analysis focuses on the transfer of information between outsiders, i.e., bond and stock investors ${ }^{5}$ Concerning this information exchange between outside investors, we may compare the present paper to the model of Harris and Raviv (1990), where debt (i) helps outside investors to acquire information $[6$ on the firm's financial health and (ii) allows outside investors to "discipline" the firm's management.7 Concerning the aspect of information revelation, the present model and the framework by Harris and Raviv (1990) have in common that the asset which reveals information is chosen by assumption. To vindicate our assumption, namely the focus on stock prices rather than equilibrium interest rates as information aggregates, we show that it is the coarseness (smoothness) of the debt's (stock's) pay-out profile which induces bond (stock) investors to invest a small (large) amount of resources into information acquisition 8

\footnotetext{
${ }^{3}$ In the absence of a unified theory of information aggregation in competitive markets, it seems best to consider both specifications. See Hellwig (1982) for a comparison of both approaches.

${ }^{4}$ See Myers (2001) for a survey which emphasizes the tax-shield-default tradeoff.

${ }^{5}$ More precisely, we will assume that the management's knowledge of the firm carries no information. An extension of our setting to a model where the management's optimal decision $\left(K^{*}, B^{*}\right)$ also carries information in itself is possible. Instead of such an extended model, where both effects coexist, we work with a reduced framework to isolate our contribution.

${ }^{6}$ In the model of Harris and Raviv (1990), it is the mere fact that a firm can meet its debt obligations at certain points in time which signals to outside investors that the firm's health is at least sufficient to pay the debt's interest. The channel through which information is revealed and the coarseness of that information therefore differ fundamentally from the present setting.

${ }^{7}$ See Calomiris and Kahn (1991) for a related model. See Admati et al. (2010), pp. 28-31, for a reflection on the governance and informational role of a firm's debt. Albagli et al. (2011) develop a model where a firm's management interacts with stock prices that aggregate investors' dispersed private information. In their model, however, firms issue no debt.

${ }^{8}$ See Dang et al. (2009) for a similar result. In a different context, they find that, due to the coarseness of the pay-out profile of debt, risk-neutral bond investors have the least incentive to acquire information.
} 
In a more remote interpretation, the present paper provides an alternative perspective on the analysis of Morris and Shin 2000, 2004), Atkeson (2000), and Angeletos and Werning (2006), who study the informational role of public signals, such as stock prices, in the context of coordination games. Suitably interpreted, these models describe a firm's debt rollover problem. In contrast to these studies, we allow for an endogenous bond yield which equalizes demand and supply in the bond market. Moreover, Morris and Shin (2000, 2004) and Angeletos and Werning (2006) study models where the quality of the agent's private information is exogenous, while the present model emphasizes that larger/deeper markets generate more informative signals since they incentivise more private information acquisition.

The rest of the paper is structured as follows. In Section 2, we lay out the model. In Section 3, we derive the main results. In a separate Section 4, we reflect on our assumptions and replace some of them to demonstrate the robustness of our findings. First, we discuss the timing of trades, which implies that it is the stock market, rather than the bond market, which aggregates information. Second, we show that the firm's strength $\theta$ can be derived from a consistent budget constraint. Third, we present a more general specification for the bond market. Finally, we show that our findings also obtain for the standard CARA-normal noisetrader equilibrium, where equity investors can condition their demands on stock prices. Section 5 offers concluding remarks.

\section{The Model}

Our model consists of the firm's management, a unit measure of stock investors, and a unit measure of bond investors. Their interaction is characterized by the following timeline:

- Period 0: The firm's management holds a prior $f$ over the unknown fundamental $\theta$. Based on these expectations, the firm decides on the optimal capital structure $(K, B)$ which minimizes the expected cost of capital $\mathbb{E}^{f}[C]=\mathbb{E}^{f}[K(\theta-p)+B R]$ subject to the revenue constraint $I=\mathbb{E}^{f}[p] K+B$. After the firm has announced a particular plan, $(K, B)$, equity investors choose their optimal information acquisition strategy. Finally, equity investors receive information and submit equity demand schedules.

- Period 1: The equity market opens and an equilibrium stock price $p$ is observed. The 


\begin{tabular}{|c|c|c|c|c|c|c|}
\hline \multicolumn{3}{|c|}{ Period 0} & \multicolumn{2}{|c|}{ Period 1} & \multicolumn{2}{|c|}{ Period 2} \\
\hline$\theta$ & $\theta_{0}$ & $\theta_{i}$ & $p$ & $R$ & $\theta$ & time \\
\hline $\begin{array}{l}\text { fundamental } \\
\text { is drawn }\end{array}$ & $\begin{array}{l}\text { initial } \\
\text { signal }\end{array}$ & $\begin{array}{l}\text { private } \\
\text { signals }\end{array}$ & price & return & $\begin{array}{l}\text { fundamental } \\
\text { is revealed }\end{array}$ & [Information] \\
\hline$(K, B)$ & \multicolumn{2}{|c|}{$\alpha(K)$} & & & & time \\
\hline \multicolumn{3}{|c|}{ capital structure information stategy } & 1 & 1 & 1 & $\longrightarrow$ \\
\hline $\begin{array}{c}\text { capital structur } \\
\text { is chosen }\end{array}$ & $\begin{array}{r}\text { inform } \\
\text { is }\end{array}$ & $\begin{array}{l}\text { stategy } \\
\text { en }\end{array}$ & $\begin{array}{c}\text { equity } K \\
\text { traded }\end{array}$ & $\begin{array}{l}\text { debt } B \\
\text { traded }\end{array}$ & $\begin{array}{c}\text { payoffs } \\
\text { materialize }\end{array}$ & [Actions] \\
\hline
\end{tabular}

debt market opens after the equity market and bonds are traded at the equilibrium yield $R(p)$.

- Period 2: The firm's unknown fundamental $\theta$ is revealed and all payoffs are realized.

Due to the correspondence between stock and bond market, we begin the description of our model with the equity market; subsequently, we introduce the bond market and, finally, we analyze the ex-ante decision of the firm's management, which is influenced by the interaction between both markets.

Fundamental and Returns The returns earned by equity and bond investors depend on the unknown strength of the firm $\theta$. In our baseline specification, this fundamental $\theta$ is exogenous and independent of the capital structure $9^{9}$ The left-hand side of Figure 1 indicates that the firm pays bond investors a return $R$ if the firm is strong enough, i.e., $\theta>0$. If the firm defaults, $\theta<0$, investors incur a loss $L$. Regarding the returns to equity investment, which are depicted on the right-hand side of Figure 1, we study a CARA-normal model, where $(\theta-p) k_{i}$ is the absolute amount of consumption goods available to an agent who invested $k_{i} ; p$ and $\theta$ are the asset's price and pay-out, respectively 10

\footnotetext{
${ }^{9}$ We show in Section 4.2 that this fundamental can be derived from the firm's resource constraint. In our baseline model, we abstract from such a resource constraint, since it links the firm's capital structure in a non-trivial way to its default probability, and the dividends paid to equity holders. In Section 4.2, we return to this omission and show that the incorporation of a resource constraint strengthens the effects that we derive for the simplified setting.

${ }^{10}$ In a different interpretation, which avoids negative prices, we discuss a CRRA-lognormal model. In this interpretation, agent $i$ derives utility from the return, $\ln \left(\frac{V}{P}\right) k_{i}=(\theta-p) k_{i}$, that she earns on her investment $k_{i}$; $p=\ln (P)$ and $\theta=\ln (V)$ are the natural logarithms of a primitive price $P>0$ and a lognormal fundamental $V>0$, respectively.
} 

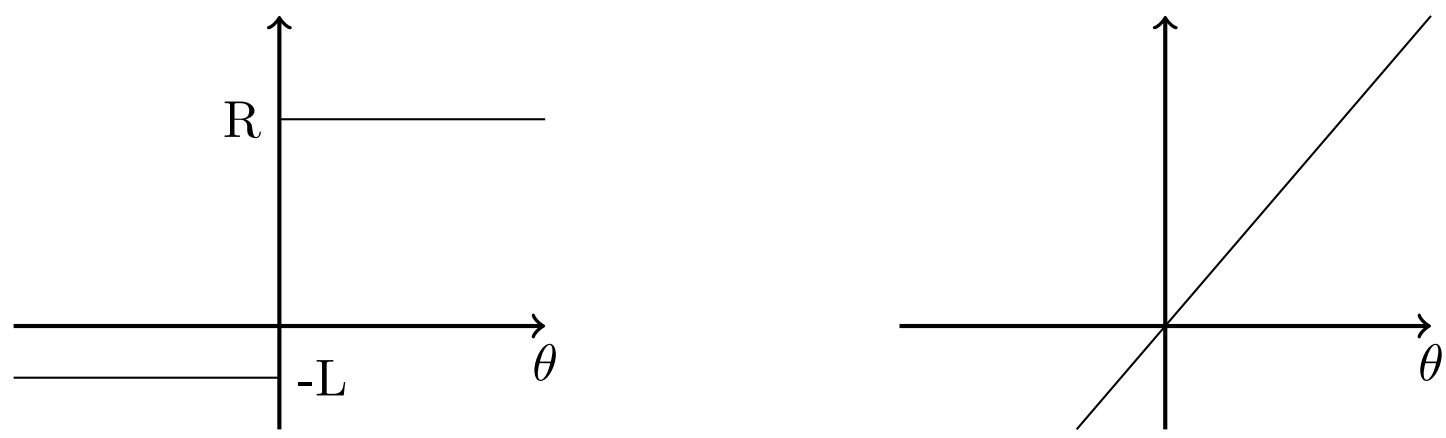

Figure 1: Net return on debt and equity as functions of $\theta$.

\subsection{The Equity Market}

Equity investors receive CARA utility from their portfolio return $x$ :

$$
U_{i}(x)=-e^{-\gamma x}
$$

where $\gamma$ represents the coefficient of absolute risk aversion.

\subsubsection{Information}

At the outset, equity investors receive a noisy signal $\theta_{0}$, which is centered around the true fundamental $\theta$. This signal $\theta_{0}$ contains a number of errors $\xi_{n}$ relative to the true state $\theta$. Formally,

$$
\theta_{0}=\theta+\sum_{n \in N} \xi_{n}
$$

where $\xi_{n} \sim \mathcal{N}\left(0, \frac{1}{|N|} \sigma^{2}\right)$ are i.i.d. errors independent of $\theta$. To improve the quality of their signal, equity investors can collect information privately to eliminate the errors contained in $\theta_{0}$. By collecting and processing the information on a certain "bit" of information $\hat{n}$, the agent recovers the value of $\xi_{\hat{n}}$. She can then correct her estimate, and obtain $\theta+\sum_{n \in N / \hat{n}} \xi_{n}$, which has a lower variance around $\theta$. If agent $i$ processes a subset $N_{i} \subseteq N$ of errors, her estimate becomes

$$
\theta_{i}=\theta+\sum_{n \in N / N_{i}} \xi_{n}
$$


Since $\theta_{i}$ dominates the initial signal $\theta_{0}$ in (2), agents will base their investment decision on $\theta_{i}$ only. That is, given $\theta_{i}$, the initial signal $\theta_{0}$ becomes fully redundant 11

All agents process information independently of each other, such that each $N_{i}$ is an independent random sample of $N$. To characterize the quality of agent $i$ 's signal, we define $\alpha_{i} \equiv \frac{\left|N_{i}\right|}{|N|}$. The posterior signal variance, after a share $\alpha_{i}$ of errors was removed from $\theta_{0}$, is therefore $\operatorname{Var} \sum_{n \in N / N_{i}} \xi_{n}=\sum_{n \in N / N_{i}} \operatorname{Var} \xi_{n}=\left(|N|-\left|N_{i}\right|\right) \frac{1}{|N|} \sigma^{2}=\left(1-\alpha_{i}\right) \sigma^{2}$. Hence, the distribution of the noisy component is $\mathcal{N}\left(0,\left(1-\alpha_{i}\right) \sigma^{2}\right)$, and for the entire signal we have $\theta_{i} \sim \mathcal{N}\left(\theta,\left(1-\alpha_{i}\right) \sigma^{2}\right)$. In the rest of the paper, we treat $\alpha_{i}$ as continuous for the sake of employing differential calculus which reduces the exposition.

The monetary cost of information acquisition is denoted by a monotonously increasing function $C\left(\alpha_{i}\right)$. Moreover, to ensure that the information acquisition decision, which we study in Section 2.1.3, has an interior solution, we assume that a complete elimination of the noise is prohibitively costly at the margin: $\lim _{\alpha_{i} \rightarrow 1} \frac{C(1)-C\left(\alpha_{i}\right)}{1-\alpha_{i}}=\infty$.

Imperfect information is the only friction in the equity market, and equity investors can choose optimal capital investment $k_{i}$ without short-selling constraints. The net return on capital investment equals $(\theta-p) k_{i}$, where $p$ is the market price. Given the private signal $\theta_{i}$, agent i's posterior distribution for $\theta$ is normal with mean $\theta_{i}$ and variance $\left(1-\alpha_{i}\right) \sigma^{2}$. Recalling

\footnotetext{
${ }^{11}$ To see that $\operatorname{Pr}\left(\theta \mid \theta_{i}, \theta_{0}\right)=\operatorname{Pr}\left(\theta \mid \theta_{i}\right)$ we note that $\operatorname{Pr}\left(\theta \mid \theta_{i}, \theta_{0}\right)=\frac{\operatorname{Pr}\left(\theta_{i}, \theta_{0} \mid \theta\right) \operatorname{Pr}(\theta)}{\int_{[0,1]} \operatorname{Pr}\left(\theta_{i}, \theta_{0} \mid \theta\right) \operatorname{Pr}(\theta) d \theta}=$ $\frac{\operatorname{Pr}\left(\theta_{i} \mid \theta\right) \operatorname{Pr}\left(\sum_{n \in N_{i}} \xi_{n}=\theta_{0}-\theta_{i} \mid \theta\right) \operatorname{Pr}(\theta)}{\int_{[0,1]} \operatorname{Pr}\left(\theta_{i} \mid \theta\right) \operatorname{Pr}\left(\sum_{n \in N_{i}} \xi_{n}=\theta_{0}-\theta_{i} \mid \theta\right) \operatorname{Pr}(\theta) d \theta}$. Due to the independence of errors from $\theta$, we have $\operatorname{Pr}\left(\sum_{n \in N_{i}} \xi_{n}=\theta_{0}-\theta_{i} \mid \theta\right)=\operatorname{Pr}\left(\sum_{n \in N_{i}} \xi_{n}=\theta_{0}-\theta_{i}\right)$ and thus $\operatorname{Pr}\left(\sum_{n \in N_{i}} \xi_{n}=\theta_{0}-\theta_{i}\right)$ cancels such that $\operatorname{Pr}\left(\theta \mid \theta_{i}, \theta_{0}\right)=\frac{\operatorname{Pr}\left(\theta_{i} \mid \theta\right) \operatorname{Pr}(\theta)}{\int_{[0,1]} \operatorname{Pr}\left(\theta_{i} \mid \theta\right) \operatorname{Pr}(\theta) d \theta}=\operatorname{Pr}\left(\theta \mid \theta_{i}\right)$.
} 
(2), the investment decision is therefore: $:{ }^{12}$

$$
\begin{aligned}
k_{i}^{d}(p) \quad & =\underset{k_{i}}{\arg \max }\left\{\mathbb{E}\left[-e^{-\gamma(\theta-p) k_{i}} \mid \theta_{i}\right]\right\} \\
& =\underset{k_{i}}{\arg \max }\left\{\gamma \mathbb{E}\left[(\theta-p) k_{i} \mid \theta_{i}\right]-\frac{\gamma^{2}}{2} \operatorname{Var}\left[(\theta-p) k_{i} \mid \theta_{i}\right]\right\} \\
& =\underset{k_{i}}{\arg \max }\left\{\gamma\left(\theta_{i}-p\right) k_{i}-\frac{\gamma^{2}}{2} k_{i}^{2}\left(1-\alpha_{i}\right) \sigma^{2}\right\},
\end{aligned}
$$

and the demand function writes:

$$
k_{i}^{d}(p)=\frac{\theta_{i}-p}{\left(1-\alpha_{i}\right) \gamma \sigma^{2}} .
$$

Demand increases in the agent's signal about the fundamental $\theta_{i}$. Risk aversion makes demand less elastic, whereas the precision of the signal, $\left(\left(1-\alpha_{i}\right) \sigma^{2}\right)^{-1}$, increases elasticity.

So far, we have characterized the optimal investment decision for a given signal quality (1$\alpha_{i}$ ). To describe investor behavior fully, it remains to solve for the ex-ante optimal information acquisition strategy. This strategy, however, depends on the ex-ante distribution of prices which is endogenous. Hence, we calculate the market equilibrium for a given information acquisition strategy $\alpha$ first. In a second step, we add the information acquisition decision and solve for the overall equity market equilibrium.

\subsubsection{Equity Market Equilibrium}

The supply of the stock at $t=1$ is fixed at $K$ such that the equilibrium condition reads: $\int_{[0,1]} k_{i}^{d}(p) d i=K$. Given the individual demand function, (4), this equilibrium condition writes:

$$
\int_{[0,1]} \frac{\theta_{i}-p}{\left(1-\alpha_{i}\right) \gamma \sigma^{2}} d i=K
$$

\footnotetext{
${ }^{12}$ For simplicity we follow Hellwig (1982) and deviate from Grossman and Stiglitz (1980), in the sense that we do not allow stock investors to use the equilibrium price, which does not yet exist at the point in time when they submit their demand schedule, to make inference on the fundamental $\theta$. That is, demand writes $k_{i}^{d}(p)=\underset{k_{i}}{\arg \max }\left\{\mathbb{E}\left[-e^{-\gamma(\theta-p) k_{i}} \mid \theta_{i}\right]\right\}$ rather than $k_{i}^{d}(p)=\underset{k_{i}}{\arg \max }\left\{\mathbb{E}\left[-e^{-\gamma(\theta-p) k_{i}} \mid \theta_{i}, p\right]\right\}$. This simplification allows us to bypass the well-known fix-point problem associated with the linear price equilibrium for CARAnormal economies where agents can condition on prices. While this shortens the exposition, it is not crucial to our analysis. We illustrate this in Section 4.4, where we show that the same results obtain for the alternative setting of Grossman and Stiglitz (1976, 1980), Green (1975), Hellwig (1980), and Angeletos and Werning (2006) in which equity investors can condition on prices.
} 
In the symmetric equilibrium, where $\alpha_{i}=\alpha$ for all $i \sqrt{13}$ we thus have:

$$
\int_{[0,1]} \theta_{i} d i-p=(1-\alpha) \gamma \sigma^{2} K
$$

and the equilibrium price equals the average estimate of the fundamental, less a fraction of the stock supply:

$$
p=\theta_{p}-(1-\alpha) \gamma \sigma^{2} K
$$

where $\theta_{p}=\int_{[0,1]} \theta_{i} d i$ is the average estimate in the population of traders. Moreover, (6) reflects that, given $\theta_{p}$, increases in stock supply and risk aversion reduce the price while increased information acquisition increases prices. Recalling the definition of $\theta_{i}$ in (3) and the fact that $N_{i}$ is sampled from $N$ independently of $i, 14$ we have the following equivalence:

$$
\begin{aligned}
\theta_{p} & =\int_{[0,1]} \theta_{i} d i=\int_{[0,1]}\left(\theta+\sum_{n \in N / N_{i}} \xi_{n}\right) d i \\
& =\theta+\sum_{n \in N} \xi_{n}-\int_{[0,1]} \sum_{n \in N_{i}} \xi_{n} d i=\theta+\sum_{n \in N} \xi_{n}-\frac{\left|N_{j}\right|}{|N|} \sum_{n \in N} \xi_{n}=\theta+\sum_{n \in N} \xi_{n}-\alpha \sum_{n \in N} \xi_{n} \\
& =\theta+(1-\alpha) \sum_{n \in N} \xi_{n}
\end{aligned}
$$

such that the average estimate $\theta_{p}$, and thus the observable variable $p+(1-\alpha) \gamma \sigma^{2} K$, are normally distributed around the true value of the fundamental: $\theta_{p} \sim \mathcal{N}\left(\theta,(1-\alpha)^{2} \sigma^{2}\right) .15$ Put differently, the market aggregates dispersed private information in the sense that prices are more informative than any individual signal $\theta_{i}$ alone, i.e., in the symmetric equilibrium where $\alpha_{i}=\alpha$, we have $\theta_{i} \sim \mathcal{N}\left(\theta,(1-\alpha) \sigma^{2}\right)$ and $(1-\alpha)^{2}<1-\alpha<11^{16}$ To close the equity market equilibrium, it remains to characterize the ex-ante information acquisition strategy $\alpha_{i}$.

\subsubsection{Equilibrium in Information Acquisition}

Agent $i$ chooses the signal's informativeness $\alpha_{i}$ to maximize her ex-ante utility. To obtain a first-order condition which allows to show that an increase in the equity market's size, $K$, leads to more information acquisition in equilibrium, it is convenient to express the ex-ante utility function in terms of means and variances. In Appendix A, we show that, given the firm's stock

\footnotetext{
${ }^{13}$ See section 2.1 .3 .

${ }^{14}$ That is, each given error $\xi_{n}$ is removed from an agent's private signal with probability $\frac{\left|N_{i}\right|}{|N|}$

${ }^{15} \operatorname{Var} \theta_{p}=\operatorname{Var}\left((1-\alpha) \sum_{n \in N} \xi_{n}\right)=(1-\alpha)^{2} \sigma^{2}$.

${ }^{16}$ Recall that agents cannot remove all errors from their signals, i.e., $\alpha \in(0,1)$.
} 
supply $K$, ex-post asset demand (4), and the distribution of equilibrium prices implied by (6), the agent's ex-ante choice of an optimal information acquisition strategy, $\alpha_{i}$, can be written as:

$$
\min _{\alpha_{i}} \mathbb{E} e^{-\frac{\left(\theta_{i}-p\right)^{2}}{2\left(1-\alpha_{i}\right) \sigma^{2}}+\gamma C\left(\alpha_{i}\right)} .
$$

In Appendix B, we characterize the comparative statics of information acquisition implied by (8):

Lemma 1. Increases in the equity market's depth $|K|$ raise private information acquisition, $\alpha(|K|)$, and the price signal's precision $\frac{1}{(1-\alpha(|K|))^{2} \sigma^{2}}$.

Lemma 1 describes how the announcement of different capital plans $(K, B)$ influences the price signal's precision and therefore the strength of the informational externality ${ }^{17}$

\subsection{Debt Market}

Analogous to the equity market, the bond market has a unit measure of CARA investors, and prices are determined by market clearing 18 The debt holders' return on the portfolio equals $b_{j} \rho(\theta)$, where $\rho(\cdot)$ is the net return on debt. If the true fundamental, $\theta$, is greater than 0 , implying that the firm is solvent, the net return is $R$. If the true fundamental is less than 0 , debt holders get the fire-sale/liquidation value incurring a loss $L$ :

$$
\rho(\theta)= \begin{cases}R & \text { if } \theta \geq 0 \\ -L & \text { if } \theta<0 .\end{cases}
$$

Demand $b_{j}^{d}(\cdot)$ for bonds solves the utility maximization problem conditional on the available information:

$$
\begin{aligned}
b_{j}^{d}(R) \quad & =\underset{b_{j}}{\arg \max } \mathbb{E}\left[-e^{-\gamma b_{j}(R) \rho(\theta)} \mid p\right] \\
& =\underset{b_{j}}{\arg \max }\left(-\operatorname{Pr}(\theta \geq 0 \mid p) e^{-\gamma R b_{j}}-\operatorname{Pr}(\theta<0 \mid p) e^{\gamma L b_{j}}\right) .
\end{aligned}
$$

That is, agents rely on the stock price signal to calculate the bankruptcy probability $1-\pi_{p}$, which depends on the unknown value of the fundamental. Let us denote the firms' survival

\footnotetext{
${ }^{17}$ Note that Lemma 1 allows for negative values of $K$ (equity buy-out) and implies that the effect on information acquisition is symmetric. In equilibrium, the optimal capital structure always involves positive levels of $K$, as we show in Lemma 4 .

${ }^{18}$ We provide a more general specification of the bond market in Section 4.3 .
} 
probability conditional on the price signal by $\operatorname{Pr}(\theta \geq 0 \mid p) \equiv \pi_{p}$. Thus

$$
\mathbb{E}\left[-e^{-\gamma \rho(\theta) b_{j}}\right]=-\pi_{p} e^{-\gamma R b_{j}}-\left(1-\pi_{p}\right) e^{\gamma L b_{j}} .
$$

We solve the first-order condition

$$
\pi_{p} \gamma R e^{-\gamma R b_{j}}-\left(1-\pi_{p}\right) \gamma L e^{\gamma L b_{j}}=0
$$

to obtain the demand function

$$
b_{j}^{d}(R)=\frac{1}{\gamma(R+L)} \ln \frac{\pi_{p} R}{\left(1-\pi_{p}\right) L} .
$$

The demand for bond decreases in risk aversion $(\gamma)$, the loss due to liquidation $(L)$, and the conditional probability of bad outcome $\left(1-\pi_{p}\right)$. The influence of $R$ is twofold: its increase implies higher returns on debt, on the one hand, but higher risks, on the other.

\subsubsection{Debt Market Equilibrium}

For a given supply of bonds, $B$, the debt markets' equilibrium condition writes:

$$
\begin{aligned}
& \int_{j \in[0,1]} b_{j}^{d}(R)=B \\
& \Leftrightarrow \frac{1}{\gamma(R+L)} \ln \frac{\pi_{p} R}{\left(1-\pi_{p}\right) L}=B \\
& \Leftrightarrow \ln \frac{R}{L}+\ln \frac{\pi_{p}}{1-\pi_{p}}=\gamma B(R+L) .
\end{aligned}
$$

From (11) we have the implicit expression for the equilibrium return on debt:

$$
\ln R=\gamma B(R+L)+\ln L-\ln \frac{\pi_{p}}{1-\pi_{p}} .
$$

Figure 2 illustrates that the equilibrium condition $(12)$ has two solutions $R_{1}$ and $R_{2}$. Where the lower interest rate equilibrium, $R_{1}$, is stable while the high interest rate equilibrium, $R_{2}$, is unstable 1900 In what follows, we focus on the stable equilibrium. Regarding the stable

\footnotetext{
${ }^{19}$ It is possible to check that increases in the rate of return increase (reduce) demand in the low (high) interest equilibrium. Hence, the low (high) interest equilibrium is Walrasian stable (unstable). See Samuelson (1941), pp. 102-106, for a discussion of the Walrasian market mechanism.

${ }^{20}$ If the price signal is negative and sufficiently low, or the bond supply is sufficiently high, the bond market may not clear at all. That is, the ex-ante plan $(K, B)$ of the firm, may be ex-post infeasible. This possibility, could be incorporated in the firm's ex-ante optimization problem. It would, however, be unrelated to the informational spill-over which we emphasize in this paper, and we omit it therefore.
} 


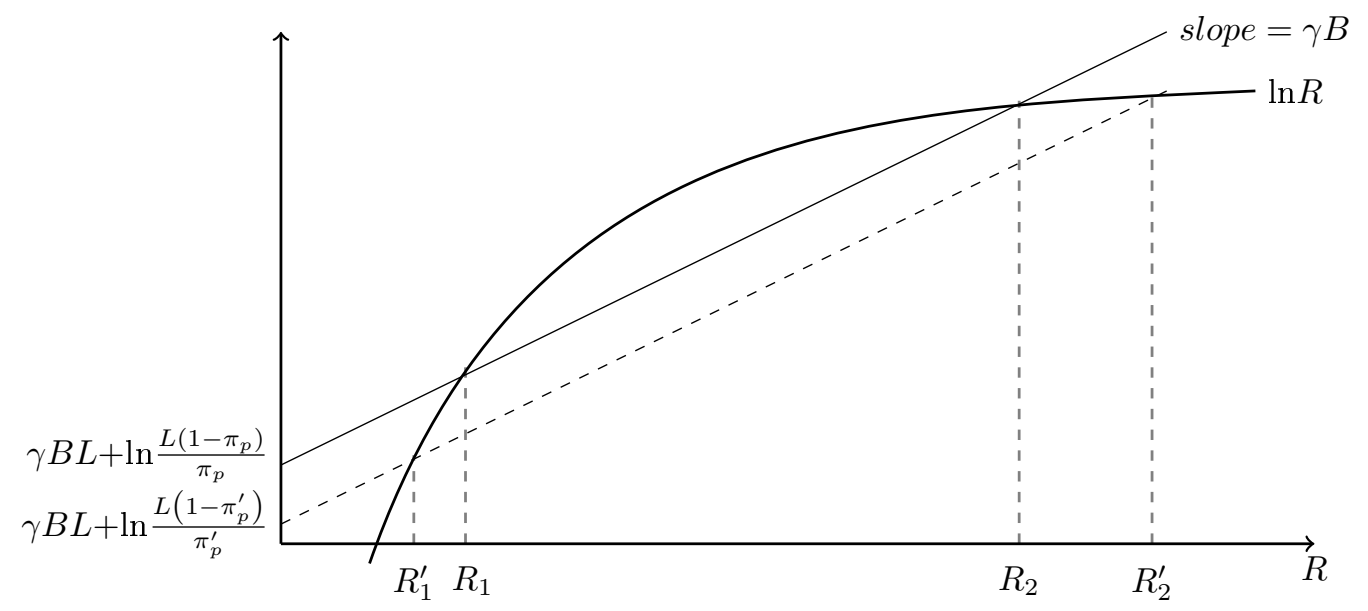

Figure 2: Debt market equilibria: for a financially stable firm $(\theta>0)$, increases in the stock price signal's precision $(\alpha)$ increase the inferred survival probability $\left(\pi_{p}^{\prime}>\pi_{p}\right)$ and reduce the interest rate $\left(R_{1}^{\prime}<R_{1}\right)$ in the stable equilibrium.

low-interest equilibrium, we immediately obtain

Lemma 2. The stable equilibrium rate of return $R$ decreases in the firm's survival probability $\pi_{p}$, and increases in the loss $L$, debt supply $B$ and risk aversion $\gamma$.

\subsubsection{Effect of the Stock Price Signal}

We recall that increases in stock investors' information acquisition $\alpha$ make the price signal $\theta_{p}=\theta+(1-\alpha) \sum_{n \in N} \xi_{n}$ more informative. Consequently, we have the following

Proposition 1. The cost of debt decreases (increases) in the amount of acquired information $\alpha$ when the fundamental $\theta$ is positive (negative).

Proof. For the stable equilibrium we have $\frac{\partial R}{\partial \pi}<0$ and therefore $\frac{\partial R}{\partial \pi} \frac{\partial \pi}{\partial \alpha}=\frac{\partial R}{\partial \pi} \phi\left(\frac{\theta}{(1-\alpha) \sigma}+\frac{\sum_{n \in N} \xi_{n}}{\sigma}\right) \frac{\theta}{(1-\alpha)^{2} \sigma} \gtreqless 0$ if $\theta \gtreqless 0$.

Figure 2 illustrates that, if $\theta>0$, increases in the stock investors' information acquisition reduce borrowing costs.

\section{Capital Structure}

In this section, we study how the informational spill-over relates to the choice of an optimal capital structure. We analyze the general equilibrium, where agents choose their information acquisition strategy as described in Section 2.1.3, such that the informativeness of the price signal varies with different capital structures. In this setting, every capital structure choice 
implies a distinct informational environment. This endogeneity induces the firm's management to issue more equity and less debt than it would if the informational spill-over was exogenous. That is, an optimizing firm chooses its capital structure to amplify the price-signal's precision, which reduces its expected borrowing costs.

The firm's management is assumed to be risk-neutral. Moreover, it requires external financing $I>0$ which it can attract by selling equity/shares $K$ and debt $B$. The firm announces its ex-ante optimal capital structure $K, B$ at $t=0$, before markets open. That is, the firm will choose its optimal capital structure subject to the anticipated informational interaction between both markets described above. We study a management that believes with certainty that the firm will not default $(\theta>0)$ and place no further restrictions on beliefs. The expectation operator associated with the management's beliefs is denoted $\mathbb{E}^{f}[]$, where the superscript $f$ refers to the firm. 21

\subsection{Optimal Capital Structure with Endogenous Information}

The firm minimizes expected capital costs $C=\left(\mathbb{E}^{f}[\theta]-\mathbb{E}^{f}[p]\right) K+\mathbb{E}^{f}[R] B$ subject to the financing constraint $I$ and the financial market equilibrium. In particular, the firm chooses its capital structure in anticipation of the positive relation between the market depth $K$ and the informativeness of the price signal described in Lemma 1. Taking into account the equilibria in bond and equity market, the firm's optimization problem reads:

$$
\min _{K, B} C\left(K, \mathbb{E}^{f}[p], \mathbb{E}^{f}[R], \alpha\right)=\min _{K, B} \mathbb{E}^{f}[(\theta-p) K+R B]
$$

subject to:

$$
\begin{cases}\text { revenue constraint } & \mathbb{E}^{f}[p] K+B=I \\ \text { equilibrium information acquisition } & \alpha=\alpha(K) \\ \text { equity market equilibrium } & p=\theta_{p}-(1-\alpha) \gamma \sigma^{2} K, \\ \text { debt market equilibrium } & \ln R=\gamma B(R+L)+\ln \frac{L\left(1-\pi_{p}\right)}{\pi_{p}},\end{cases}
$$

\footnotetext{
${ }^{21}$ In equilibrium, stock and bond investors can invert the optimal plan $\left(K^{*}, B^{*}\right)$ and solve for the management's prior $\mathbb{E}^{f}[\theta]$. As we point out in the introduction, we assume that the management's prior is uninformative (to investors), to separate the impact of information spill-overs from stock prices from the signalling effects (Footnote 5 that were studied in previous papers.
} 
where the conditional survival probability for the firm is $\pi_{p}=\Phi\left(\frac{\theta_{p}}{(1-\alpha) \sigma}\right)$. To study 13 we reduce it to a problem in $K$ :

$$
\min _{K} C(K, p, R, \alpha), \quad \text { s.t. } \quad p=p(K, \alpha), \quad R=R\left(I-\mathbb{E}^{f}[p] K, \alpha\right), \quad \alpha=\alpha(K),
$$

which yields the main result:

Proposition 2. In the endogenous information setting the firm issues more equity and less debt to internalize the informational externality that stock prices exert on bond yields.

Proof. The first order condition:

$$
\frac{d C}{d K}=\frac{\partial C}{\partial K}+\frac{\partial C}{\partial \alpha} \frac{\partial \alpha}{\partial K}
$$

shows that the capital cost minimization problem consists of a direct effect $\frac{\partial C}{\partial K}$, and an indirect effect $\frac{\partial C}{\partial \alpha} \frac{\partial \alpha}{\partial K}$, which represents how the informational content of prices changes with the capital structure. In Appendix $\mathrm{C}$, we characterize the properties of the direct effect, $\frac{\partial C}{\partial K}$, which describes the capital structure problem for any exogenously given information structure $\alpha$. In particular, we show that, if the revenue requirement $I$ is sufficiently high, there exist values $K^{*}>0$ and $B^{*}>0$, for which $\frac{\partial C}{\partial K}=0$; and $K^{*}>0$ and $B^{*}>0$ represent a global cost minimum. To prove that the firm issues more equity and less debt in the endogenous information optimum, such that $K^{* *}>K^{*}$, it remains to show that to show that $\frac{\partial C}{\partial \alpha} \frac{\partial \alpha}{\partial K}<0$ at $K^{*}$. That is, if $\left.\frac{d C}{d K}\right|_{K^{*}}=0+\frac{\partial C}{\partial \alpha} \frac{\partial \alpha}{\partial K}<0$, then the firm issues more equity and less debt to internalize the informational externality. Regarding this externality, we note that

$$
\frac{\partial C}{\partial \alpha} \frac{\partial \alpha}{\partial K}=\left(\frac{\partial C}{\partial \mathbb{E}^{f}[p]}-\frac{\partial C}{\partial \mathbb{E}^{f}[R]} \frac{\partial \mathbb{E}^{f}[R]}{\partial B} K\right) \frac{\partial \mathbb{E}^{f}[p]}{\partial \alpha} \frac{\partial \alpha}{\partial K}+\frac{\partial C}{\partial \alpha} \frac{\partial \alpha}{\partial K}
$$

Differentiation of the constraints in (14) yields a more explicit expression:

$$
\frac{\partial C}{\partial \alpha} \frac{\partial \alpha}{\partial K}=\mathbb{E}^{f}\left[1+R+\frac{\partial R}{\partial B} B\right] K \gamma \sigma^{2} \frac{\partial \alpha}{\partial K}+B \mathbb{E}^{f}\left[\frac{\partial R}{\partial \alpha} \frac{\partial \alpha}{\partial K}\right]
$$

From Proposition 1, Lemma 4, and Lemma 2, we know that (i) $K^{*}>0$ such that information acquisition is increasing in $K: \frac{\partial \alpha}{\partial K}>0$, and (ii) the expected equilibrium rate of return on debt decreases in the price signal's precision: $\frac{\partial R}{\partial \alpha}<0$. Thus we can estimate the sign of (17), at the exogenous information optimum $K^{*}$ where $\frac{\partial C}{\partial K}=0$, as:

$$
-\underbrace{\left(1+\mathbb{E}^{f}\left[R+\frac{\partial R}{\partial B} B^{*}\right]\right) \gamma \sigma^{2}}_{+} \frac{\partial \alpha}{\partial K} K^{*}+\underbrace{\mathbb{E}^{f}\left[\frac{\partial R}{\partial \alpha} \frac{\partial \alpha}{\partial K}\right] B^{*}}_{-}<0,
$$

where the negative sign in 18 is ensured if the financing requirement $I$ is sufficiently high, such that, by Lemma 4, $B^{*}$ is positive. Hence, at the exogenous information optimum we have $\frac{d C^{* *}}{d K}\left(K^{*}\right)<0$, and an increase in $K$ from $K^{*}$ towards $K^{* *}$ decreases capital costs.

That is, 18 reflects that if the firm takes into account that every capital structure is associated with a particular information structure, it issues more equity and less debt since a 
marginal increment in equity (i) increases information acquisition and thus allows to sell the equity in place, $K^{*}$, at a higher price and (ii) reduces borrowing costs since debt holders rely more heavily on the price signal, which is on average positive since the expected fundamental is positive $\mathbb{E}^{f}[\theta]>0$. More precisely, the first term in $[18),\left(1+\mathbb{E}^{f}\left[R+\frac{\partial R}{\partial B} B^{*}\right]\right) \gamma \sigma^{2} \frac{\partial \alpha}{\partial K} K^{*}$, reflects that increases in capital supply raise information acquisition, which increases investor demand such that a given stock supply $K^{*}$ can be sold at higher prices. The term $\mathbb{E}^{f}\left[R+\frac{\partial R}{\partial B} B^{*}\right]$ reflects that this increase in prices allows to reduce debt, which, in turn, reduces borrowing costs on the remaining debt $B^{*}$. The second term, $\mathbb{E}^{f}\left[\frac{\partial R}{\partial \alpha} \frac{\partial \alpha}{\partial K}\right] B^{*}$ is the information externality from stock price signal to bond yield which increases in the stock market's size $K$.

\section{Discussion}

A number of our previous assumptions were made to facilitate a parsimonious exposition. In this section, we reflect on our assumptions and replace some of them to demonstrate the robustness of our findings. First, we discuss the assumption that the stock market rather than the bond market is the main source of public information. Second, we show that the firm's dividend $\theta$, and thus the survival probability $\pi(\theta>0 \mid p)$, can be derived from a consistent budget constraint. Third, we present a more general specification for the bond market. Finally, we show that our finding that the stock price's informational content increases with the market size $|K|$ also obtains for the standard noise-trader equilibrium of Grossman and Stiglitz 1980, 1976) and Hellwig (1980), where equity investors can condition on prices.

\subsection{Direction of the Spillover}

As it is, the model structure immediately implies that the stock market signal influences the bond market equilibrium. In principle, it is possible to construct an alternative model where bond investors research the firm's financial health and stock investors use the equilibrium yield to infer the firm's fundamental. Alternatively, we could study the intermediate case where both $P$ and $R$ carry information. While such an analysis is possible, we believe that the current specification, where the stock price signal influences bond yields, is likely the most relevant one: (i) empirically we find that, even though many firms operate with a capital structure, where the value of the debt far exceeds the value of equity, it is the stock price and not the yield of 


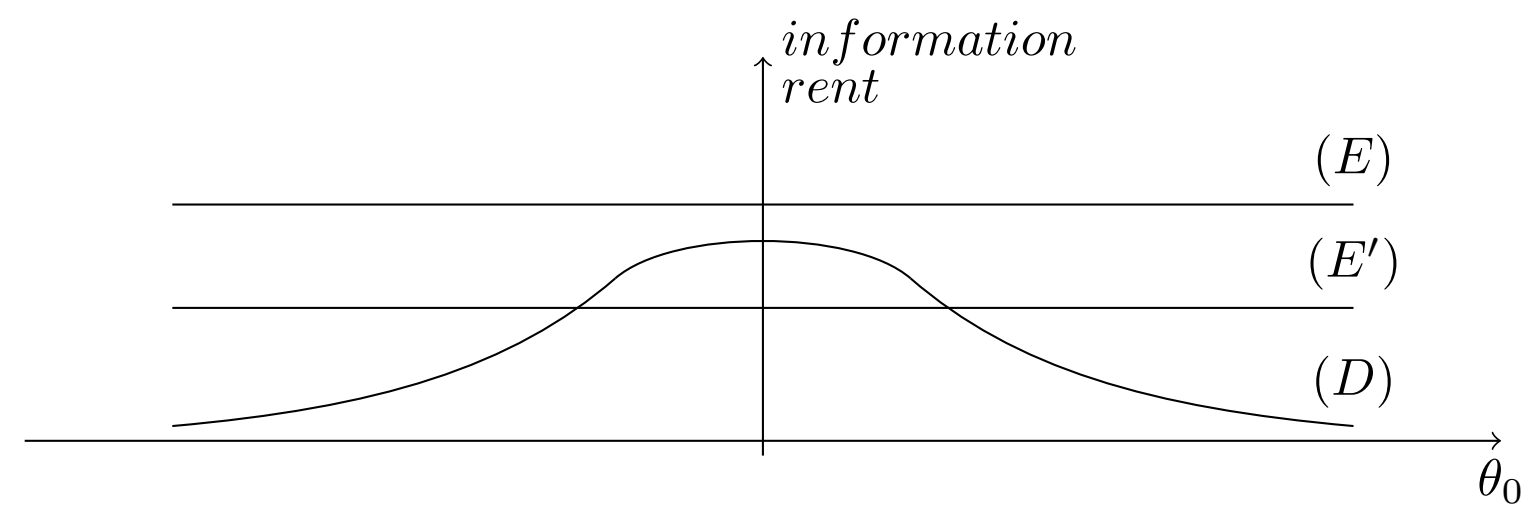

Figure 3: Incentive to acquire information in the debt market $(D)$ and in the equity market $(E)$ as a function of the prior $\theta_{0}$.

a bond (of some reference maturity) that is published most prominently in the media. ${ }^{22}$ (ii) Technically, we find that the stock price tends to be more informative than the bond yield in the following sense: ${ }^{23}$

Lemma 3. The incentive to acquire information for equity holders, and therefore the informativeness of the stock price signal, is independent of the firm's expected financial health $\theta_{0}$. The incentive to acquire information for bond investors is hump-shaped and vanishes as $\theta_{0}$ becomes either large or small.

Proof. The first part follows immediately from the fact that the first order condition (27), which describes the equity investor's incentive to acquire information, $\alpha$, is independent of the firm's expected fundamental $\theta_{0}$. The stock price signal's quality, $\frac{1}{(1-\alpha)^{2} \sigma^{2}}$, is therefore also independent of $\theta_{0}$. The proof that bond investors have no incentive to acquire information, if the firm appears to be either very healthy or seems to be bankrupt with near certainty, relies on the coarseness of the pay-out profile of bonds. Unlike stock investors, whose payout at the margin depends on the precise value of the fundamental $\theta$, bond investors are only interested to asses whether the firm is solvent $(\theta>0)$ or insolvent $(\theta<0)$. The formal argument is given in Appendix A.3.

Broadly interpreted, Lemma 3 indicates that stock prices, unlike the bond yields, are a "reliable" source of information "at all times". In terms of modeling assumptions, we therefore find that a sufficiently large $\theta_{0}$, which is accompanied by a small fixed cost of information acquisition, ensures that it is optimal for bond investors to rely purely on the stock price signal even though they could, in principle, acquire information on their own.

\footnotetext{
${ }^{22}$ See Footnote 2

${ }^{23}$ In a different context, Dang et al. (2009) derive a related result which indicates that the pay-out profile of bonds has the consequence that risk-neutral bondholders have less incentive to acquire information than investors who own other assets. While this "information insensitivity" property makes debt attractive in the model of Dang et al. (2009), it makes debt unattractive in the present model.
} 


\subsection{The Firm's Resource Constraint}

To simplify the exposition in the main text, we treated the firm's dividend $\theta$ as independent of the capital structure. In this appendix, we modify our model to incorporate a consistent budget constraint.

The firm's financial strength $Y$ allows it to service the debt $R B$ and to pay a dividend of $\theta$ per share:

$$
Y=K \theta+R B \quad \Leftrightarrow \quad \theta=\frac{Y}{K}-\frac{R B}{K} .
$$

The equilibrium price for a share is thus: ${ }^{24}$

$$
p=\mathbb{E}[\theta]-\gamma(1-\alpha) \sigma^{2} K
$$

In Appendix D.1, we show that the informational externality, which induces firms to issue more equity and less debt, also obtains once dividends $\theta$, and the firm's survival probability $\pi(\theta>0 \mid p)$, depend on the interest rate as in 19 .

\subsection{Alternative Bond Market}

In our baseline model, bond demand is derived from a CARA utility function. However, for our result to obtain we only require that bond demand is given by a continuously differentiable function:

$$
B^{D}=B^{D}(\pi(\theta>0 \mid p), R), \quad \frac{\partial B^{D}}{\partial \pi}>0, \quad \frac{d B^{D}}{d R}=\frac{\partial B^{D}}{\partial \pi} \frac{\partial \pi}{\partial \theta} \frac{\partial \theta}{\partial R}+\frac{\partial B^{D}}{\partial R}>0,
$$

where $\theta$ is defined as in (19). The assumptions regrading the demand function's derivatives indicate that for every given interest rate, demand increases in the firm's survival probability. Moreover, increases in the rate of return increase bond demand 25 Put differently, we assume that the increased return $R$ outweighs the decrease $\left(\frac{\partial B^{D}}{\partial \pi} \frac{\partial \pi}{\partial \theta} \frac{\partial \theta}{\partial R}<0\right)$ in the probability with

\footnotetext{
${ }^{24}$ Note that $\mathrm{R}=\mathrm{R}(\mathrm{p})$, where $\mathrm{p}$ is known at the time when trade happens (ex-ante, the incentive to research theta is not affected by $\mathrm{R}(\mathrm{p}) \mathrm{B}$, since investors only search for the part $\frac{Y}{K}$ which is unknown at the point in time when they trade (conditional on $\mathrm{p}$, which fully determines $\mathrm{R}(\mathrm{p})$ ). Moreover, note that $\theta=\frac{Y}{K}-\frac{R B}{K}$ vanishes in the difference $\theta_{i}-p$, in the information acquisition problem (25). Hence, for the alternative budget constraint, the positive relation between the informativeness of stock price and equity supply $K$ can be derived as before.

${ }^{25}$ This second assumption is equivalent to the assumption that the bond market equilibrium is Walrasian stable.
} 
which $\theta>0$. In equilibrium, where $B^{S}=B$, we have

$$
B^{D}(\pi, R)=B \quad \Leftrightarrow \quad R=R(\pi(\theta>0 \mid p), B)>0, \quad \frac{\partial R}{\partial B}>0, \quad \frac{\partial R}{\partial \pi}<0 .
$$

Where the signs of $\frac{\partial R}{\partial B}$, and $\frac{\partial R}{\partial \pi}$ follow from 21]. Again, we show in Appendix D.1 that the informational externality, which induces firms to issue more equity and less debt, obtains if (21) and (22) characterize bond demand.

\subsection{Conditioning on Prices}

Our result relies crucially on the fact that the stock price signal's precision increases with the size $K$ of the stock market. In our baseline model, we derived this result for a stock market where, for simplicity, we did not allow equity investors to condition their demands on stock prices. In Appendix 4.4, we show that this result also obtains in the standard ${ }^{26}$ linear CARA-normal noise trader equilibrium where equity investors can condition their demand functions on the informational content of prices. That is, the demand function $k_{i}=\arg \max \left\{\mathbb{E}\left[-e^{-\gamma(\theta-p) k_{i}} \mid \theta_{i}, p\right]\right\}$ also incorporates the price signal $p$ and not just the private signal $\theta_{i}$.

\section{Conclusion}

The present paper provides a simple equilibrium model in which a firm's stock price serves as a costless rating device for investors who buy its debt. The main motivation for our analysis lies in the observation that stock investors, as opposed to rating agencies, which rely on fees from the firm's they rate, have no incentive to misreport their information on the firm's financial health. That is, they do not buy stocks at inflated prices to mislead bond investors.

To study the spill-over from stock price signals to bond yields, we have assumed the perspective of a firm that minimizes its capital cost subject to the informational connection between bond and stock market. In a first step, we have shown that, for a given information structure, firms that are financially strong benefit from an informative stock price which communicates the financial strength of the firm more clearly to bond investors who rely on the stock price signal to infer the firms' default probability. In a second step, we endogenize the strength of the informational spill-over. In an economy where equity investors can choose how much information to acquire, we find that an increase in the amount of equity issued incentivises stock investors to

\footnotetext{
${ }^{26}$ See Grossman and Stiglitz (1976, 1980), Hellwig (1980), and Angeletos and Werning (2006).
} 
research the firm more carefully, which increases the stock price signal's precision. Hence, the strength of the informational spill-over varies with different capital structures. Consequently, the firm can, through its capital structure, internalize part of the informational externality that stock prices exert on bond yields. Firms with a strong fundamental will therefore issue more equity and less debt, than they would if the informational spill-over did not exist, to generate stock price signals which communicate its strong financial position on average more clearly to bond investors.

In one interpretation, the firm's capital cost minimization problem is simply an exposition device, which helps to illustrate how a firm's stock price interacts with its borrowing costs through its informational content. In a different interpretation, the current paper complements the literature that studies a firm's optimal capital structure in models with default and asymmetric information. It suggests the role of competitive markets as information aggregation devices as another aspect, which shapes a firm's financing decisions. 


\section{A Appendix}

To show that ex-ante utility of an agent $i$ writes $\mathbb{E} U_{i}=\mathbb{E}\left[-\exp \left(-\frac{\left(\theta_{i}-p\right)^{2}}{2\left(1-\alpha_{i}\right) \sigma^{2}}+\gamma C\left(\alpha_{i}\right)\right)\right]$ as in (8), we note that by the law of iterated expectations we have:

$$
\mathbb{E}\left[U_{i}\right]=\mathbb{E}\left[\left(\mathbb{E} U_{i} \mid \theta_{i}\right)\right]
$$

where the equity holder's utility in expectation, after a signal $\theta_{i}$ was received (ex interim), writes:

$$
\begin{aligned}
& \mathbb{E}\left[U \mid \theta_{i}\right]=\mathbb{E}\left[U\left((\theta-p) k_{i}^{d}(p)-C\left(\alpha_{i}\right)\right) \mid \theta_{i}\right] \\
& =\mid(4) \mathbb{E}\left[U\left((\theta-p) \frac{\theta_{i}-p}{\left(1-\alpha_{i}\right) \gamma \sigma^{2}}-C\left(\alpha_{i}\right)\right) \mid \theta_{i}\right] .
\end{aligned}
$$

By (3), the true $\theta$ is normally distributed around the signal $\theta_{i}, \theta \sim \mathcal{N}\left(\theta_{i},\left(1-\alpha_{i}\right) \sigma^{2}\right)$. Recalling the utility function (1), we rewrite 23 as:

$$
\begin{aligned}
& \mathbb{E}\left[U_{i} \mid \theta_{i}\right]= \\
& =-\exp \left(-\gamma \mathbb{E}\left[(\theta-p) \frac{\theta_{i}-p}{\left(1-\alpha_{i}\right) \gamma \sigma^{2}}-C\left(\alpha_{i}\right) \mid \theta_{i}\right]+\frac{\gamma^{2}}{2} \operatorname{Var}\left[\frac{(\theta-p)\left(\theta_{i}-p\right)}{\left(1-\alpha_{i}\right) \gamma \sigma^{2}} \mid \theta_{i}\right]\right) \\
& =-\exp \left(-\frac{\left(\theta_{i}-p\right)^{2}}{\left(1-\alpha_{i}\right) \sigma^{2}}+\frac{\gamma^{2}}{2}\left(\frac{\theta_{i}-p}{\left(1-\alpha_{i}\right) \gamma \sigma^{2}}\right)^{2}\left(1-\alpha_{i}\right) \sigma^{2}+\gamma C\left(\alpha_{i}\right)\right) \\
& =-\exp \left(-\frac{\left(\theta_{i}-p\right)^{2}}{2\left(1-\alpha_{i}\right) \sigma^{2}}+\gamma C\left(\alpha_{i}\right)\right)
\end{aligned}
$$

If we take expectations with regard to $\theta_{i}-p$, then (24) becomes (8), which is what we needed to show. To work with (24), we note that it follows from (3) and (6) that

$$
\begin{aligned}
& \theta_{i}-p=\theta_{i}-\left(\theta_{p}-(1-\alpha) \gamma \sigma^{2} K\right) \\
& =\theta+\sum_{n=N / N_{i}} \xi_{n}-\left(\theta+(1-\alpha) \sum_{n \in N} \xi_{n}-\gamma(1-\alpha) \sigma^{2} K\right) \\
& =\sum_{n=N / N_{i}} \xi_{n}-(1-\alpha) \sum_{n \in N} \xi_{n}+\gamma(1-\alpha) \sigma^{2} K
\end{aligned}
$$


We therefore have $\operatorname{Var}\left(\theta_{i}-p\right)=\left(\alpha_{i} \beta^{2}+\alpha^{2} \beta_{i}\right) \sigma^{2}$, where $\beta=1-\alpha$ and $\beta_{i}=1-\alpha_{i}$, and the difference $\theta_{i}-p$ is normally distributed with $\theta_{i}-p \sim \mathcal{N}\left(\gamma \beta \sigma^{2} K ;\left(\alpha_{i} \beta^{2}+\alpha^{2} \beta_{i}\right) \sigma^{2}\right)$.

\section{B Proof of Lemma 1}

$$
\min _{\alpha_{i}} \mathbb{E} e^{-\frac{\left(\theta_{i}-p\right)^{2}}{2 \beta_{i} \sigma^{2}}+\gamma C\left(\alpha_{i}\right)}=\min _{\alpha_{i}} \mathbb{E} e^{-\frac{\alpha_{i} \beta^{2}+\alpha^{2} \beta_{i}}{2 \beta_{i}} Z^{2}+\gamma C\left(\alpha_{i}\right)}
$$

where, the difference $\theta_{i}-p$ is normally distributed, $\theta_{i}-p \sim \mathcal{N}\left(\gamma \beta \sigma^{2} K ;\left(\alpha_{i} \beta^{2}+\alpha^{2} \beta_{i}\right) \sigma^{2}\right)$, with $\beta=1-\alpha$ and $\beta_{i}=1-\alpha_{i}$. Accordingly, $z^{2} \equiv \frac{\left(\theta_{i}-p\right)^{2}}{\left(\alpha_{i} \beta^{2}+\alpha^{2} \beta_{i}\right) \sigma^{2}}$ follows a noncentral $\chi^{2}$-distribution with one degree of freedom and non-centrality parameter $\frac{\left(\gamma \beta \sigma^{2} K\right)^{2}}{\left(\alpha_{i} \beta^{2}+\alpha^{2} \beta_{i}\right) \sigma^{2}}=\frac{(\gamma \beta \sigma K)^{2}}{\alpha_{i} \beta^{2}+\alpha^{2} \beta_{i}} \equiv \lambda$. We can therefore use the moment generating function, $\mathbb{E} e^{b z^{2}}=(1-2 b)^{-\frac{1}{2}} e^{\frac{b \lambda}{1-2 b}}$, for the noncentral $\chi^{2}$ distribution 27 , with $b=-\frac{\alpha_{i} \beta^{2}+\alpha^{2} \beta_{i}}{2 \beta_{i}}$, and $\lambda=\frac{(\gamma \beta \sigma K)^{2}}{\alpha_{i} \beta^{2}+\alpha^{2} \beta_{i}}$, to rewrite problem 25 as:

$$
\min _{\alpha_{i}}(1-2 b)^{-\frac{1}{2}} e^{\frac{\lambda b}{1-2 b}+\gamma C\left(\alpha_{i}\right)}, \quad b=b\left(\alpha_{i}\right) .
$$

The first-order condition:

$$
(1-2 b)^{-\frac{3}{2}} b^{\prime} e^{\frac{\lambda b}{1-2 b}+\gamma C\left(\alpha_{i}\right)}+(1-2 b)^{-\frac{1}{2}} e^{\frac{\lambda b}{1-2 b}+\gamma C\left(\alpha_{i}\right)}\left(\frac{\left(\lambda b^{\prime}+\lambda^{\prime} b\right)(1-2 b)+2 \lambda b b^{\prime}}{(1-2 b)^{2}}+\gamma C^{\prime}\left(\alpha_{i}\right)\right)=0,
$$

rewrites as:

$$
(1-2 b)^{-\frac{3}{2}} e^{\frac{\lambda b}{1-2 b}+\gamma C\left(\alpha_{i}\right)}\left(b^{\prime}+\lambda^{\prime} b+\frac{\lambda b^{\prime}}{1-2 b}+\gamma C^{\prime}\left(\alpha_{i}\right)(1-2 b)\right)=0 .
$$

The interior optimum ${ }^{28}$ described in 27 allows to study the comparative statics of information acquisition:

Lemma 1: Increases in the equity market's depth $|K|$ raise private information acquisition, $\alpha(|K|)$, and the price signal's precision $\frac{1}{(1-\alpha(|K|))^{2} \sigma^{2}}$.

Proof. Denote the left-hand side of (27) by $F\left(\alpha_{i}, K^{2}\right)$. In equilibrium, equation (27) is an identity, implying that $F\left(\alpha_{i}, K^{2}\right) \equiv 0$. The effect of the market depth $|K|$, on the equilibrium

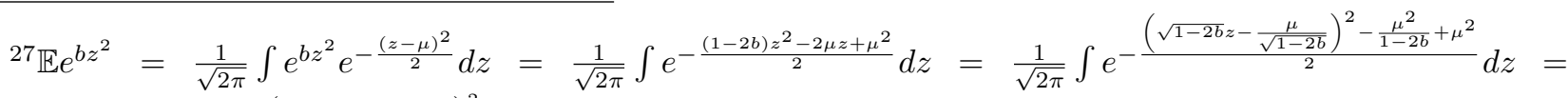

$$
\begin{aligned}
& \frac{1}{\sqrt{1-2 b} \sqrt{2 \pi}} \int e^{\frac{\mu^{2} b}{1-2 b}} e^{-\frac{\left(\sqrt{1-2 b} z-\frac{\mu}{\sqrt{1-2 b}}\right)^{2}}{2}} d(\sqrt{1-2 b} z)=\frac{1}{\sqrt{1-2 b}} e^{\frac{\mu^{2} b}{1-2 b}} \text {. }
\end{aligned}
$$

${ }^{28}$ Our assumption that a complete elimination of errors is prohibitively costly, $\lim _{\alpha_{i} \rightarrow 1} \frac{C(1)-C\left(\alpha_{i}\right)}{1-\alpha_{i}}=\infty$, ensures that (27) has an interior solution. 
$\alpha_{i}$, now follows from the implicit function theorem:

$$
\frac{d \alpha_{i}}{d K^{2}}=-\frac{\partial F\left(\alpha_{i}, K^{2}\right)}{\partial K^{2}} / \frac{\partial F\left(\alpha_{i}, K^{2}\right)}{\partial \alpha_{i}}>0 .
$$

To see that (28) is positive we note that: the numerator is negative since it follows from (27) that $29 \frac{\partial F\left(\alpha_{i}, K^{2}\right)}{\partial K^{2}}=(1-2 b)^{-\frac{3}{2}} e^{\frac{\lambda b}{2 b-1}+\gamma C\left(\alpha_{i}\right)} \frac{\lambda b^{\prime}}{K^{2}(1-2 b)}+\underbrace{F\left(\alpha_{i}, K^{2}\right)}_{=0} \frac{\lambda b^{\prime}}{K^{2}(1-2 b)}<0$, since $b^{\prime}=-\frac{\beta^{2}}{2 \beta_{i}^{2}}<$ 0. That the denominator of $(28)$ is positive follows from the second-order condition, which implies that $\frac{\partial F\left(\alpha, K^{2}\right)}{\partial \alpha}>0$ at the optimal $\alpha$. Therefore, we have $\frac{d \alpha}{d K^{2}}>0$, and increases in $K^{2}$ result in a reduced variance of the private signal $\left(1-\alpha_{i}\right) \sigma^{2}$, i.e., more information acquisition. To show that the inverse of the price signal's variance, i.e., the precision $\frac{1}{(1-\alpha)^{2} \sigma^{2}}$, increases with $\alpha$, we recall that $\alpha \in(0,1)$ is ensured by the cost function $C(\alpha)$. Consequently, we have $\frac{d\left(\frac{1}{(1-\alpha(K))^{2} \sigma^{2}}\right)}{d K}>0$.

\section{Optimal Capital Structure: The "Direct Effect"}

In this appendix, we characterize the direct effect $\frac{\partial C}{\partial K}$. The firm minimizes expected capital costs

$$
\min _{K, B} C\left(K, \mathbb{E}^{f}[p], \mathbb{E}^{f}[R], \alpha\right)=\min _{K, B}\left(\mathbb{E}^{f}[\theta]-\mathbb{E}^{f}[p]\right) K+\mathbb{E}^{f}[R] B
$$

subject to the market system:

$$
\begin{cases}\text { revenue constraint } & \mathbb{E}^{f}[p] K+B=I \\ \text { equilibrium information acquisition } & \alpha=\alpha(K) \\ \text { equity market equilibrium } & p=\theta_{p}-(1-\alpha) \gamma \sigma^{2} K, \\ \text { debt market equilibrium } & \ln R=\gamma B(R+L)+\ln \frac{L\left(1-\pi_{p}\right)}{\pi_{p}},\end{cases}
$$

where the conditional survival probability for the firm is $\pi_{p}=\Phi\left(\frac{\theta_{p}}{(1-\alpha) \sigma}\right)$. By substituting for the level of debt $B=I-\mathbb{E}^{f}[p] K$, Problem 29 becomes a problem in $K$ alone:

$$
\min _{K} C\left(K, \mathbb{E}^{f}[p], \mathbb{E}^{f}[R], \alpha\right), \quad \text { s.t. } \quad p=p(K, \alpha), \quad R=R(\underbrace{I-\mathbb{E}^{f}[p] K}_{B}, \alpha) .
$$

Where $p(K, \alpha)$ is the solution for the equity market equilibrium (6), and $R(B, \alpha)$ is the stable bond market equilibrium (11). The first-order condition for the cost minimizing $K^{*}$, which

\footnotetext{
${ }^{29}$ Note $\lambda$ is linear in $K^{2}$; thus, $\frac{\partial \lambda}{\partial K^{2}}=\frac{\lambda}{K^{2}}$.
} 
leaves out the indirect effect $\frac{\partial C}{\partial \alpha} \frac{\partial \alpha}{\partial K}$ is therefore: Differentiation of constraint 30, yields: ${ }^{30}$

$$
\frac{\partial C}{\partial K}=2(1-\alpha) \gamma \sigma^{2} K \mathbb{E}^{f}\left[1+R+\frac{\partial R}{\partial B} B\right]-\mathbb{E}^{f}[\theta] \mathbb{E}^{f}\left[R+\frac{\partial R}{\partial B} B\right]=0 .
$$

Before we show that (32) locates one global and potentially several local minima, we characterize certain properties of the solution $\left(K^{*}, B^{*}\right)$ implied by 32$)$ :

Lemma 4. For any positive revenue $I$, the optimal level of equity $K^{*}$ is positive. If the financing requirement $I$ is sufficiently large, the firm always relies on both markets to raise funds and we have $B^{*}>0$ and $K^{*}>0$ at the optimum.

Proof. See Appendix D

While Lemma 4 establishes that the firm issues positive quantities of debt and equity at the interior optimum if the financing requirement $I$ is sufficiently large, it leaves open the question whether there are corner solutions. To complete the characterization of the solution, we note that all possible minima (local and global) are finite:

Lemma 5. The capital cost minimization problem has a finite global minimum.

Proof. See Appendix D

\section{Proof of Lemmas 4 and 5}

We recall the target revenue constraint $\mathbb{E}^{f}[p] K+B=I$, the equity market equilibrium $p=$ $\theta_{p}-\beta \gamma \sigma^{2} K$ with $\beta=1-\alpha$, and the debt market equilibrium $\ln R=\gamma B(R+L)+\ln \frac{L\left(1-\pi_{p}\right)}{\pi_{p}}$ to rewrite

$$
\frac{\partial C}{\partial K}=\mathbb{E}^{f}[\theta]-\mathbb{E}^{f}[p]+\left(\frac{\partial C}{\partial \mathbb{E}^{f}[p]}-\frac{\partial C}{\partial \mathbb{E}^{f}[R]} \frac{\partial \mathbb{E}^{f}[R]}{\partial B} K\right) \frac{\partial \mathbb{E}^{f}[p]}{\partial K}-\frac{\partial C}{\partial \mathbb{E}^{f}[R]} \frac{\partial \mathbb{E}^{f}[R]}{\partial B} \mathbb{E}^{f}[p]=0(
$$

as:

$$
\begin{aligned}
=\mathbb{E}^{f}[\theta]-\mathbb{E}^{f}[p]-\mathbb{E}^{f}[R] \mathbb{E}^{f}[p]+\left(-K-\mathbb{E}^{f}[R] K-B \frac{\partial \mathbb{E}^{f}[R]}{\partial B} K\right)\left(-\beta \gamma \sigma^{2}\right)-B \frac{\partial \mathbb{E}^{f}[R]}{\partial B} \mathbb{E}^{f}[p] \\
=\mathbb{E}^{f}[\theta]-\mathbb{E}^{f}[p](1+R)+\beta \gamma \sigma^{2}\left(1+R+\frac{\partial \mathbb{E}^{f}[R]}{\partial B} B\right) K-B \frac{\partial \mathbb{E}^{f}[R]}{\partial B} \mathbb{E}^{f}[p] \\
=\mathbb{E}^{f}[\theta]+\left(\beta \gamma \sigma^{2} K-\mathbb{E}^{f}[p]\right)\left(1+R+\frac{\partial \mathbb{E}^{f}[R]}{\partial B} B\right)
\end{aligned}
$$

\footnotetext{
${ }^{30}$ See Appendix $\mathrm{D}$ for the derivation.
} 


$$
=\mathbb{E}^{f}[\theta]+\left(2 \beta \gamma \sigma^{2} K-\mathbb{E}^{f}[\theta]\right)\left(1+\mathbb{E}^{f}[R]+\frac{\partial \mathbb{E}^{f}[R]}{\partial B} B\right)=0
$$

Proof of Lemma 4 First, to prove that $K^{*}$ is positive if $I>0$, we show that the first order condition (34) is violated for any $K \leq 0$. Namely, we show that the derivative is strictly negative at $K \leq 0$, that is, issuing more equity reduces capital costs.

We rearrange the terms of (34) as follows:

$$
\frac{d C}{d K}=2 \beta \gamma \sigma^{2} K \mathbb{E}^{f}\left[1+R+\frac{\partial R}{\partial B} B\right]-\mathbb{E}^{f}[\theta] \mathbb{E}^{f}\left[R+\frac{\partial R}{\partial B} B\right]=0 .
$$

The first term $2 \beta \gamma \sigma^{2} K \mathbb{E}^{f}\left[1+R+\frac{\partial R}{\partial B} B\right]$ is non-positive for $K \leq 0$ since we know (i) from Lemma 2 (debt market equilibrium) that $R>0$ and $\frac{\partial R}{\partial B}>0$, and, (ii) the revenue constraint implies that $B \geq I>0$ for $K \leq 0$. The second term of $[35), \mathbb{E}^{f}[\theta] \mathbb{E}^{f}\left[R+\frac{\partial R}{\partial B} B\right]$, is positive as long as $K \leq 0$ since $\mathbb{E}^{f}[\theta]>0$. Hence, $\frac{d C}{d K}$ is negative for $K \leq 0$, implying that the cost minimizing $K^{*}$ is positive.

Next, we show that $B^{*}>0$, if the financing requirement $I$ is sufficiently high. To show this, we note that it follows from the revenue constraint $I=\mathbb{E}^{f}[p] K+B$, the price function $\mathbb{E}^{f}[p]=$

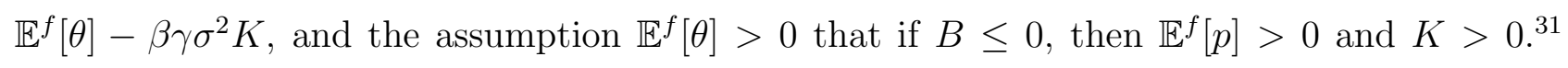
It therefore follows from 35 that we have $\left.\frac{d C}{d K}\right|_{B=0, K=\frac{I}{E p}}=2 \beta \gamma \sigma^{2} \frac{I}{\mathbb{E}^{f}[p]} \mathbb{E}^{f}[1+R]-\mathbb{E}^{f}[\theta] \mathbb{E}^{f}[R]$. One can now show that for every given expectation $\mathbb{E}^{f}[\theta]>0$, a sufficiently large financing requirement $I$ ensures $\frac{d C}{d K} \mid B=0, K=\frac{I}{E p}>0$. That is, a reduction in $K$, which implies an increase in $B$, reduces capital costs such that $B^{*}>0$.

Proof of Lemma 5 We use the target revenue constraint to eliminate $B$ from (34):

$$
\begin{aligned}
& =\mathbb{E}^{f}[\theta]+\left(2 \beta \gamma \sigma^{2} K-\mathbb{E}^{f}[\theta]\right)\left(1+\mathbb{E}^{f}\left[R+\frac{\partial R}{\partial B}\right]\left(I-K \mathbb{E}^{f}[p]\right)\right) \\
& =\mathbb{E}^{f}[\theta]+\left(2 \beta \gamma \sigma^{2} K-\mathbb{E}^{f}[\theta]\right)\left(1+\mathbb{E}^{f}\left[R+\frac{\partial R}{\partial B}\right]\left(I-K\left(\mathbb{E}^{f}[\theta]-\beta \gamma \sigma^{2} K\right)\right)\right) .
\end{aligned}
$$

In equation $36, K^{3}$ is the largest exponent, and it has a non-negative coefficient $2\left(\beta \gamma \sigma^{2}\right)^{2} \frac{\partial R}{\partial B} \geq$ 0 . Derivative $(36)$ is therefore positive as $K \rightarrow+\infty \sqrt[32]{32}$ Moreover, we know from Lemma 4 that

\footnotetext{
${ }^{31}$ By contradiction, we find that an allocation where $\mathbb{E}^{f}[p]<0$ and $K<0$, such that $I=\mathbb{E}^{f}[p] K>0$, is impossible since we have assumed that $\mathbb{E}^{f}[\theta]>0$ such that $\mathbb{E}^{f}[p]=\mathbb{E}^{f}[\theta]-\beta \gamma \sigma^{2} K>0$ for $K<0$ which contradicts the initial assumption that $\mathbb{E}^{f}[p]>0$.

${ }^{32}$ If $2\left(\beta \gamma \sigma^{2}\right)^{2} \frac{\partial R}{\partial B}=0$ then the largest exponent is $K$ with a coefficient that is positive.
} 
(36) is negative for $K \leq 0$. The continuity of 36 then ensures that it must equal 0 at (at least) one positive finite $K^{*}$, which is a solution to the first order condition.

\section{D.1 Informational Externality with Budget Constraint}

In this appendix we derive the informational externality for the modified specifications given in Sections 4.24.3. The capital cost problem:

$$
\min _{K, B} C=\min _{K, B} E[(\theta-p) K+R B] \quad \text { s.t. } \quad I=p K+B
$$

is equivalent to

$$
\begin{aligned}
& \min _{K, B} C=\min _{K, B} E\left[\left(\theta-\mathbb{E}[\theta]+\gamma(1-\alpha) \sigma^{2} K\right) K+R B\right] \quad \text { s.t. } \quad I=p K+B \\
& \min _{K, B} C=\min _{K, B} E\left[\gamma(1-\alpha) \sigma^{2} K^{2}+R B\right] \quad \text { s.t. } \quad I=p K+B .
\end{aligned}
$$

From (39), we calculate:

$$
\frac{d C}{d K}=E\left[\frac{\partial C}{\partial K}+\frac{\partial C}{\partial(1-\alpha)} \frac{\partial(1-\alpha)}{\partial K}\right]
$$

where the externality $\frac{\partial C}{\partial(1-\alpha)} \frac{\partial(1-\alpha)}{\partial K}$ induces firms to issue more equity and less debt since 33

$$
\begin{aligned}
\frac{\partial C}{\partial(1-\alpha)} \frac{\partial(1-\alpha)}{\partial K}=\quad & E\left[\left[K^{2} \frac{\partial c}{\partial(1-\alpha)}\left(1+R+\frac{\partial R}{\partial B} B\right)+\frac{\partial R}{\partial(1-\alpha)} B\right] \frac{\partial(1-\alpha)}{\partial K}\right. \\
& \left.-\left(R+\frac{\partial R}{\partial B} B\right) \frac{\partial \theta}{\partial(1-\alpha)} \frac{\partial(1-\alpha)}{\partial K} K\right]<0, \quad c:=\gamma(1-\alpha) \sigma^{2} .
\end{aligned}
$$

The first term $E\left[\left[K^{2} \frac{\partial c}{\partial(1-\alpha)}\left(1+R+\frac{\partial R}{\partial B} B\right)+\frac{\partial R}{\partial(1-\alpha)} B\right]\right] \frac{\partial(1-\alpha)}{\partial K}<0$ in $\sqrt{42}$ is identical to the effect 18 from the baseline model. The second term, $E\left[-\left(R+\frac{\partial R}{\partial B} B\right) \frac{\partial \theta}{\partial(1-\alpha)} \frac{\partial(1-\alpha)}{\partial K} K\right]<0$, originates for the budget constraint (19). It is negative since one can show that $R>0$, $\frac{\partial R}{\partial B}>0, \frac{\partial(1-\alpha)}{\partial K}<0$, and $\mathbb{E}\left[\frac{\partial \theta}{\partial(1-\alpha)}\right]=\mathbb{E}\left[-\frac{\frac{\partial R}{\partial(1-\alpha)} B}{K}\right]<0$ since $E^{f}[\theta]>0$. Hence, the reduction in borrowing costs allows to increase dividends, which increases the price at which shares sell $\frac{\partial \theta}{\partial(1-\alpha)} \frac{\partial(1-\alpha)}{\partial K} K>0$. In turn, the firm can sell fewer bonds, which reduces interest expenses $-R$. Finally, the reduction in borrowing reduces interest costs on the outstanding debt $-\frac{\partial R}{\partial B} B$.

Adding a budget constraint, (19), therefore amplifies the conclusion that the firm issues more equity to internalize the informational externality that stock prices have on bond yields.

\footnotetext{
${ }^{33}$ Note that $R=R(\pi, B)=R\left(\pi((1-\alpha)(K)), I-\theta K+c K^{2}\right)$.
} 


\section{References}

Admati, A. R., DeMarzo, P. M., Hellwig, M. F., and Pfleiderer, P. (2010). Fallacies, irrelevant facts, and myths in the discussion of capital regulation: Why bank equity is not expensive. Preprint Max Planck Institute for Research on Collective Goods, (42):1-60. 7

Albagli, E., Hellwig, C., and Tsyvinski, A. (2011). Information aggregation, investment, and managerial incentives. NBER Working Paper, (17330):1-36. 7

Angeletos, G.-M. and Werning, I. (2006). Crises and prices: Information aggregation, multiplicity, and volatility. American Economic Review, 96(5):1720-1736. 1, 12, 26, 34

Atkeson, A. (2000). Discussion on morris and shin. In NBER Macroeconomics Annual, pages 161-170. ed. Bernanke, Ben S. and Kenneth Rogoff, Cambridge, MIT Press. 1

Calomiris, C. W. and Kahn, C. M. (1991). The role of demandable debt in structuring optimal banking arrangements. American Economic Review, 81(3):497-513. 7

Dang, T. V., Gorton, G., and Holmstrm, B. (2009). Ignorance and the optimality of debt for the provision of liquidity. Working Paper, pages 1-43. 8, 23

Green, J. R. (1975). Information efficiency and equilibrium. HIER Working Paper, (284):1-29. 1. 12

Grossman, S. and Stiglitz, J. (1976). Information and competitive price systems. American Economic Review Papers and Proceedings, 66(2):246-253. 1, 12, 4, 26, 34

Grossman, S. and Stiglitz, J. (1980). On the impossibility of informationally efficient markets. American Economic Review, 70(3):393-408. 1, 12, 4, 26, 34

Harris, M. and Raviv, A. (1990). Capital structure and the informational role of debt. Journal of Finance, 45(2):321-349. 1, 6

Harris, M. and Raviv, A. (1991). The theory of capital structure. Journal of Finance, 46(1):297355. 1, 1

Hellwig, M. (1980). On the aggregation of information in competitive markets. Journal of Economic Theory, 22:477-498. 1, 12, 4, 26, 34 
Hellwig, M. (1982). Rational expectations equilibrium with conditioning on past prices: A mean variance example. Journal of Economic Theory, 26(2):279-312. 3, 12

Morris, S. and Shin, H. S. (2000). Rethinking multiple equilibria in macroeconomic modeling. In NBER Macroeconomics Annual, pages 139-161. ed. Bernanke, Ben S. and Kenneth Rogoff, Cambridge, MIT Press. 1

Morris, S. and Shin, H. S. (2004). Coordination risk and the price of debt. European Economic Review, 48(1):133-153. 1

Myers, S. C. (2001). Capital structure. Journal of Economic Perspectives, 15(2):81-102. 4

Raiffa, H. and Schlaifer, R. (2000). Applied Statistical Decision Theory. Wiley Classics Library. 35

Samuelson, P. A. (1941). The stability of equilibrium: Comparative statics and dynamics. Econometrica, 9(2):97-120. 19 


\section{A Separate Appendix}

\section{A.1 Conditioning on Prices}

In the present appendix, we show that our analysis also obtains in the standard $\sqrt{34}$ linear CARAnormal noise trader equilibrium where equity investors can condition their demand functions on the informational content of prices. Regarding information, agents receive noisy private signals $\theta_{i}=\theta+\sigma_{x} \xi_{i}$, where idiosyncratic noise $\xi_{i}$ is i.i.d. across the population and $\xi_{i} \sim \mathcal{N}(0,1)$. Moreover, agents hold an uninformative uniform prior. Noise trader activity, $\sigma_{\varepsilon} \varepsilon, \varepsilon \sim \mathcal{N}(0,1)$, ensures that prices are only partially revealing. To characterize the market price signal, we proceed in three steps. First, we guess that there exists a linear price function, $p=\eta_{1} \theta+$ $\eta_{2} \varepsilon+c$. Regarding $\theta$, this function is informationally equivalent to a signal $Z \equiv \frac{p-c}{\eta_{1}}=\theta+\frac{\eta_{2}}{\eta_{1}} \varepsilon$, which reveals the true fundamental with precision $\alpha_{z}=\frac{\eta_{1}^{2}}{\eta_{2}^{2}}$. Second, given this price function, we characterize individual demands based on the information $\theta_{i}, Z$ and calculate the market equilibrium. Finally, we determine the ratio $\frac{\eta_{1}^{2}}{\eta_{2}^{2}}$ as $\alpha_{x}^{2} \frac{1}{\gamma^{2} \sigma_{\varepsilon}^{2}}$. That is, price signal $Z$ indeed partially reveals the true fundamental $\theta$ with precision $\alpha_{z}=\alpha_{x}^{2} \frac{1}{\gamma^{2} \sigma_{\varepsilon}^{2}}$.

Demand Agents choose their optimal demands $k_{i}$ for the asset to maximize expected CARA utility

$$
\begin{aligned}
k_{i} \quad & =\underset{k_{i}}{\arg \max }\left\{\mathbb{E}\left[-e^{-\gamma(\theta-p) k_{i}} \mid \theta_{i}, Z\right]\right\} \\
& =\underset{k_{i}}{\arg \max }\left\{e^{\gamma \mathbb{E}\left[(\theta-P) k_{i} \mid \theta_{i}, Z\right]-\frac{\gamma^{2}}{2} \operatorname{Var}\left[(\theta-p) k_{i} \mid \theta_{i}, Z\right]}\right\} \\
& =\underset{k_{i}}{\arg \max }\left\{\gamma\left(\frac{\alpha_{x}}{\alpha} \theta_{i}+\frac{\alpha_{z}}{\alpha} Z-p\right) k_{i}-\frac{\gamma^{2}}{2} k_{i}^{2} \frac{1}{\alpha}\right\}, \quad \alpha=\alpha_{x}+\alpha_{z}
\end{aligned}
$$

and the individual demand function writes:

$$
k_{i}^{d}=\frac{\frac{\alpha_{x}}{\alpha} \theta_{i}+\frac{\alpha_{z}}{\alpha} Z-p}{\alpha^{-1} \gamma}
$$

\footnotetext{
${ }^{34}$ See Grossman and Stiglitz (1976, 1980), Hellwig (1980), and Angeletos and Werning (2006).

${ }^{35}$ See Raiffa and Schlaifer (2000), p. 250, for the standard results on prior and posterior distributions of normally distributed variables which we use in the following.
} 
Equilibrium Aggregate supply $K^{S}=K+\sigma_{\varepsilon} \varepsilon$, is unobservable and distorted by noise-trader activity $\varepsilon \sim \mathcal{N}(0,1){ }^{36}$ From 42 , we find that aggregate demand $K^{D}$ is:

$$
K^{D}=\int_{[0,1]} k_{i} d i=\frac{\frac{\alpha_{x}}{\alpha} \theta+\frac{\alpha_{z}}{\alpha} Z-p}{\alpha^{-1} \gamma} .
$$

Equilibrium requires that:

$$
K^{D}=K^{S} \quad \Leftrightarrow \quad \frac{\frac{\alpha_{x}}{\alpha} \theta+\frac{\alpha_{z}}{\alpha} Z-P}{\alpha^{-1} \gamma}=K+\sigma_{\varepsilon} \varepsilon
$$

To close the argument, we now resubstitute $Z=\frac{p-c}{\eta_{1}}$ and calculate the ratio $\beta=\frac{\eta_{1}}{\eta_{2}}$. First, we solve (44) for $p$ to obtain:

$$
p=\frac{\alpha_{x}}{\alpha-\frac{\alpha_{z}}{\eta_{1}}} \theta-\frac{\gamma \sigma_{\varepsilon}}{\alpha-\frac{\alpha_{z}}{\eta_{1}}} \varepsilon+\frac{-\alpha_{z} \frac{c}{\eta_{1}}-K \gamma}{\alpha-\frac{\alpha_{z}}{\eta_{1}}} .
$$

Comparison of (45) with our initial guess, $p=\eta_{1} \theta+\eta_{2} \varepsilon+c$, indicates that $\eta_{1}, \eta_{2}$ must satisfy:

$$
\eta_{1}=\frac{\alpha_{x}}{\alpha-\frac{\alpha_{z}}{\eta_{1}}}, \quad \eta_{2}=-\frac{\gamma \sigma_{\varepsilon}}{\alpha-\frac{\alpha_{z}}{\eta_{1}}} ; \quad \alpha=\alpha_{x}+\alpha_{z}
$$

To determine $\eta_{1}, \eta_{2}$, we define $\beta=\frac{\eta_{1}}{\eta_{2}}$ and note that $\alpha_{z}=\frac{\eta_{1}^{2}}{\eta_{2}^{2}}$, which follows from the definition $Z=\frac{p-c}{\eta_{1}}=\theta+\frac{\eta_{2}}{\eta_{1}} \varepsilon$. Rewriting 46 now yields $\beta=-\alpha_{x} \frac{1}{\gamma \sigma_{\varepsilon}}$, and, therefore $\alpha_{z}=\beta^{2}=\alpha_{x}^{2} \frac{1}{\gamma^{2} \sigma_{\varepsilon}^{2}}$.

That is, the agent who observes $p$, and knows the coefficients of the model, can calculate $Z=\frac{p-c}{\eta_{1}}=\theta+\frac{\eta_{2}}{\eta_{1}} \varepsilon$, which is equivalent to:

$$
Z=\theta+\alpha_{x}^{-1} \gamma \sigma_{\varepsilon} \varepsilon
$$

Accordingly, $Z$ reveals the true fundamental $\theta$ with precision $\alpha_{z}=\frac{\alpha_{x}^{2}}{\gamma^{2} \sigma_{\varepsilon}^{2}}$. Finally, we note that $\eta_{1}=1, \eta_{2}=-\sqrt{\alpha_{z}}$, and $c=\frac{-K \gamma}{\alpha}$. Accordingly, $p=\theta-\sqrt{\alpha_{z}} \varepsilon-\frac{K \gamma}{\alpha}$.

\section{A.2 Information Acquisition}

Agents' use their information $\theta_{i}, Z$ to infer $\theta$ with posterior mean $\mathbb{E}\left[\theta \mid \theta_{i}, Z\right]=\psi_{i} \equiv \frac{\alpha_{x, i}}{\alpha_{i}} \theta_{i}+\frac{\alpha_{z}}{\alpha_{i}} Z$ and precision $\alpha_{i}=\alpha_{x, i}+\alpha_{z}$, where $\alpha_{z}=\frac{\alpha_{x}^{2}}{\gamma^{2} \sigma_{\varepsilon}^{2}}$. To calculate ex-ante utility of $\alpha_{x, i}$, we write:

$$
\begin{aligned}
& \mathbb{E}[U]=\mathbb{E}\left[-e^{-\gamma k_{i}(\theta-p)+\gamma C\left(\alpha_{x, i}\right)}\right]=\mathbb{E}_{\psi_{i}, p}\left[\mathbb{E}\left[-e^{-\gamma k_{i}(\theta-p)+\gamma C\left(\alpha_{x, i}\right)} \mid \psi_{i}\right]\right] \\
& =\mathbb{E}_{\psi_{i}, p}\left[-e^{-\gamma \mathbb{E}\left[(\theta-p) k_{i} \mid \psi_{i}\right]+\frac{\gamma^{2}}{2} \operatorname{Var}\left[(\theta-p) k_{i} \mid \psi_{i}\right]+\gamma C\left(\alpha_{x, i}\right)}\right]=\mathbb{E}_{\psi_{i}, p}\left[-e^{-\gamma\left(\psi_{i}-p\right) k_{i}+\frac{\gamma^{2}}{2} \alpha_{i}^{-1} k_{i}^{2}+\gamma C\left(\alpha_{x, i}\right)}\right] .
\end{aligned}
$$

\footnotetext{
${ }^{36}$ That is, the firm's supply $K$ is known. The position of noise traders $\sigma_{\varepsilon} \varepsilon$ in the asset is unknown.

${ }^{37}$ We note that $\eta_{2}=\frac{\eta_{1}}{\beta}$ such that $\eta_{2}=-\frac{\gamma \sigma_{\varepsilon}}{\alpha-\frac{\alpha_{z}}{\eta_{1}}}$ is equivalent to $\eta_{1}=-\frac{\beta \gamma \sigma_{\varepsilon}}{\alpha-\frac{\alpha_{z}}{\eta_{1}}}$. At the same time, we have $\eta_{1}=\frac{\alpha_{x}}{\alpha-\frac{\alpha_{z}}{\eta_{1}}}$. Hence, $-\frac{\beta \gamma \sigma_{\varepsilon}}{\alpha-\frac{\alpha_{z}}{\eta_{1}}}=\frac{\alpha_{x}}{\alpha-\frac{\alpha_{z}}{\eta_{1}}}$ and thus $\beta=-\alpha_{x} \frac{1}{\gamma \sigma_{\varepsilon}}$.
} 
Using (42), we can rewrite this such that the choice of an optimal information acquisition strategy $\alpha_{x, i}$ is the solution to:

$$
\min _{\alpha_{x, i}} E\left[e^{-\frac{\left(\psi_{i}-p\right)^{2}}{2 \alpha_{i}^{-1}}+\gamma C\left(\alpha_{x, i}\right)}\right], \quad C^{\prime}()>0, \quad C^{\prime \prime}()>0 .
$$

To work with 48, we recall that $p=\theta-\sqrt{\alpha_{z}} \varepsilon-\frac{K \gamma}{\alpha}$, and note:

$$
\begin{gathered}
\psi_{i}-p=\quad \frac{\alpha_{x, i}}{\alpha_{i}} \theta_{i}+\frac{\alpha_{z}}{\alpha_{i}} Z-\theta+\sqrt{\alpha_{z}} \varepsilon+\frac{K \gamma}{\alpha}=\frac{\alpha_{x, i}}{\alpha_{i}}\left(\theta+\sigma_{x, 1} \xi\right)+\frac{\alpha_{z}}{\alpha_{i}}\left(\theta \sigma_{z} \varepsilon\right)-\theta+\sqrt{\alpha_{z}} \varepsilon+\frac{K \gamma}{\alpha} \\
=\frac{\alpha_{x, i}}{\alpha_{i}} \sigma_{x, i} \xi+\frac{\alpha_{z}}{\alpha_{i}} \sigma_{z} \varepsilon+\sqrt{\alpha_{z}} \varepsilon+\frac{K \gamma}{\alpha} .
\end{gathered}
$$

Accordingly, $\left(\psi_{i}-p\right) \sim \mathcal{N}\left(\frac{K \gamma}{\alpha}, \frac{\alpha_{x, i}}{\alpha_{i}^{2}}+\left(\frac{\sqrt{\alpha_{z}}}{\alpha_{i}}+\sqrt{\alpha_{z}}\right)^{2}\right)$. As in Appendix B, we define $y_{i} \equiv \psi_{i}-p$, such that $\Gamma^{2} \equiv \frac{y_{i}^{2}}{\operatorname{Var}\left(y_{i}\right)}$ follows a non-central $\chi^{2}$ distribution with parameter $\lambda=\frac{K^{2} \gamma^{2}}{\alpha^{2}} \frac{1}{\frac{\alpha_{x, i}}{\alpha_{i}}+\left(\frac{\sqrt{\alpha_{z}}}{\alpha_{i}}+\sqrt{\alpha_{z}}\right)^{2}}$. Once we define $b=\sqrt{\alpha_{x, i}+\left(\sqrt{\alpha_{z}}+\alpha_{i} \sqrt{\alpha_{z}}\right)^{2}}$, as in Appendix B, the MGF allows to rewrite (48) as

$$
\min _{\alpha_{x, i}}(1-2 b)^{-\frac{1}{2}} e^{\frac{\lambda b}{1-2 b}+\gamma C\left(\alpha_{x, i}\right)}, \quad b=b\left(\alpha_{x, i}\right) .
$$

The evaluation of (50) is now parallel to that in Appendix $B$, and it follows from the definition of $b$ that $\frac{\partial b}{\partial \alpha_{x, i}}>0$ and hence $\frac{d \alpha_{x, i}}{d K}>0$.

\section{A.3 Incentive to acquire information in the Bond market}

To show that the bondholder's incentive to acquire information is hump shaped, we examine the ex-ante utility of a bondholder who observes $\theta_{0}=\theta+\xi$. As in the baseline model, this investor can reduce the error contained in $\theta_{0}$, i.e., she can choose the "precision" $\alpha_{i}>0$ with which she observes the firm's true financial health $\theta$ through the signal $\theta_{i}=\theta_{0}-\alpha_{i} \xi=\theta+(1-\alpha) \xi$. That is, she can choose the conditional distribution $\theta \mid \theta_{i} \sim \mathcal{N}\left(\theta_{i},\left(1-\alpha_{i}\right) \sigma\right)$. For simplicity, we set the loss $L$ which investors incur if the firm defaults to one, such that the ex-ante utility maximization problem associated with $\alpha_{i}$ writes:

$$
\max _{\alpha_{i}} \mathbb{E}\left[\mathbb{E}\left[U \mid \theta_{i}\right]\right]=\max _{\alpha_{i}}\left\{\mathbb{E}\left[-\Phi\left(\frac{\theta_{0}-\alpha_{i} \xi}{\left(1-\alpha_{i}\right) \sigma}\right) e^{-\gamma R b_{i}}-\left(1-\Phi\left(\frac{\theta_{0}-\alpha_{i} \xi}{\left(1-\alpha_{i}\right) \sigma}\right)\right) e^{\gamma b_{i}}\right]\right\}
$$

To prove the lemma, we proceed in three steps. First, we show that the expected marginal utility of information acquisition $\frac{\partial \mathbb{E}[U]}{\partial \alpha_{i}}\left(y=\theta_{0}-\alpha_{i} \xi\right)$ goes to zero for all ex-interim signal realizations $\xi$, as the "prior" $\theta_{0}$ becomes arbitrarily strong. Second, we show that ex-ante expected marginal utility of information acquisition, $\mathbb{E}\left[\frac{\partial \mathbb{E}[U]}{\partial \alpha_{i}}\left(y=\theta_{0}-\alpha_{i} \xi\right)\right]$, goes to zero as $\theta_{0}$ 
becomes arbitrarily strong. Finally, we show that the ex-ante incentive to acquire information also goes to zero as $\theta_{0}$ becomes arbitrarily weak. Taken together, we therefore find that the incentive to acquire information is hump-shaped, i.e., the incentive for bondholders to acquire information is highest for firms on the "brink of default".

Marginal Value of Information for a given signal error $\xi$ After a particular signal $\theta_{i}=\theta_{0}-\alpha_{i} \xi$ has materialized, agent $i$ chooses $b_{i}$ to maximize:

$$
\mathbb{E}\left[U \mid \theta_{i}\right]=-\Phi\left(\frac{\theta_{0}-\alpha_{i} \xi}{\left(1-\alpha_{i}\right) \sigma}\right) e^{-\gamma R b_{i}}-\left(1-\Phi\left(\frac{\theta_{0}-\alpha_{i} \xi}{\left(1-\alpha_{i}\right) \sigma}\right)\right) e^{\gamma b_{i}}
$$

Hence, the marginal change in expected utility from increases in the precision $\alpha_{i}$ is:

$$
\begin{aligned}
\frac{\partial \mathbb{E}[U]}{\partial \alpha_{i}}\left(\theta_{0}-\alpha_{i} \xi\right) & =\phi\left(\frac{\theta_{0}-\alpha_{i} \xi}{\left(1-\alpha_{i}\right) \sigma}\right) \frac{\theta_{0}-\xi}{\left(1-\alpha_{i}\right)^{2} \sigma}\left(e^{\gamma b_{i}}-e^{-\gamma R b_{i}}\right) \\
& +\left(\Phi\left(\frac{\theta_{0}-\alpha_{i} \xi}{\left(1-\alpha_{i}\right) \sigma}\right) e^{-\gamma R b_{i}} R \gamma-\left(1-\Phi\left(\frac{\theta_{0}-\alpha_{i} \xi}{\left(1-\alpha_{i}\right) \sigma}\right)\right) e^{\gamma b_{i}} \gamma\right) \frac{\partial b_{i}}{\partial \alpha_{i}}
\end{aligned}
$$

To calculate the change in ex-interim investment, $\frac{\partial b_{i}}{\partial \alpha_{i}}$, we note that agent i's bond demand is $b_{i}=\ln \left(\frac{\Phi\left(\frac{\theta_{0}-\alpha_{i} \xi}{\left(1-\alpha_{i}\right) \sigma}\right)}{1-\Phi\left(\frac{\theta_{0}-\alpha_{i} \xi}{\left(1-\alpha_{i}\right) \sigma}\right)} R\right) \frac{1}{\gamma(R+1)}$. Accordingly, we have:

$$
\frac{\partial b_{i}}{\partial \alpha_{i}}=\phi\left(\frac{\theta_{0}-\alpha_{i} \xi}{\left(1-\alpha_{i}\right) \sigma}\right)\left(\frac{1}{\Phi\left(\frac{\theta_{0}-\alpha_{i} \xi}{\left(1-\alpha_{i}\right) \sigma}\right)}+\frac{1}{1-\Phi\left(\frac{\theta_{0}-\alpha_{i} \xi}{\left(1-\alpha_{i}\right) \sigma}\right)}\right) \frac{\theta_{0}-\xi}{\left(1-\alpha_{i}\right)^{2} \sigma} \frac{1}{\gamma(R+1)} .
$$

Moreover, to show that the incentive to acquire information, i.e. (53) and (54), vanishes as $\theta_{0}$ goes to infinity, we note that, in a symmetric equilibrium where $\alpha_{i}=\alpha$, the interest rate is given by:

$$
B=\ln \left(\frac{\Phi\left(\frac{\theta_{0}-\alpha \xi}{(1-\alpha) \sigma}\right)}{1-\Phi\left(\frac{\theta_{0}-\alpha \xi}{(1-\alpha) \sigma}\right)} R\right) \frac{1}{\gamma(R+1)} \quad \Leftrightarrow \quad e^{B \gamma(R+1)}=\frac{\Phi\left(\frac{\theta_{0}-\alpha \xi}{(1-\alpha) \sigma}\right)}{1-\Phi\left(\frac{\theta_{0}-\alpha \xi}{(1-\alpha) \sigma}\right)} R
$$

Once we focus on the stable equilibrium (Footnote 19 we have $\lim _{\theta_{0} \rightarrow \infty} R=0, \lim _{\theta_{0} \rightarrow \infty} \Phi\left(\frac{\theta_{0}-\alpha \xi}{(1-\alpha) \sigma}\right)=$ 1 , and, $\lim _{\theta_{0} \rightarrow \infty} R \frac{\Phi}{1-\Phi}=e^{\gamma B}$, i.e., the net return $R$ on the risky bond goes to zero as the probability of a default vanishes. Finally we note that in a symmetric equilibrium we have $b_{i}=B$ for a unit population of investors.

With these observations in place it follows that $\lim _{\theta_{0} \rightarrow \infty} \phi\left(\frac{\theta_{0}-\alpha_{i} \xi}{\left(1-\alpha_{i}\right) \sigma}\right) \frac{\theta_{0}-\xi}{\left(1-\alpha_{i}\right)^{2} \sigma}\left(e^{\gamma b_{i}}-e^{-\gamma R b_{i}}\right)=$ $\lim _{\theta_{0} \rightarrow \infty} e^{-\left(\frac{\theta_{0}-\alpha_{i} \xi}{\left(1-\alpha_{i}\right)}\right)^{2}} \frac{\theta_{0}-\xi}{\left(1-\alpha_{i}\right)^{2} \sigma} \lim _{\theta_{0} \rightarrow \infty}\left(e^{\gamma b_{i}}-e^{-\gamma R b_{i}}\right)=0 \times\left(e^{\gamma B}-1\right)=0$ such that $(53)$, the first 
increment in utility from improved information, vanishes. Regarding the second increment, (54), we find that $\lim _{\theta_{0} \rightarrow \infty}\left(\Phi\left(\frac{\theta_{0}-\alpha_{i} \xi}{\left(1-\alpha_{i}\right) \sigma}\right) e^{-\gamma R b_{i}} R \gamma-\left(1-\Phi\left(\frac{\theta_{0}-\alpha_{i} \xi}{\left(1-\alpha_{i}\right) \sigma}\right)\right) e^{\gamma b_{i}} \gamma\right) \times \phi\left(\frac{\theta_{0}-\alpha_{i} \xi}{\left(1-\alpha_{i}\right) \sigma}\right)\left(\frac{1}{\Phi\left(\frac{\theta_{0}-\alpha_{i} \xi}{\left(1-\alpha_{i}\right) \sigma}+\right.}+\right.$ $\left.\frac{1}{1-\Phi\left(\frac{\theta_{0}-\alpha_{i} \xi}{\left(1-\alpha_{i}\right) \sigma}\right)}\right) \frac{\theta_{0}-\xi}{\left(1-\alpha_{i}\right)^{2} \sigma} \frac{1}{\gamma(R+1)}=0$ since we have $\lim _{\theta_{0} \rightarrow \infty} e^{-\left(\frac{\theta_{0}-\alpha_{i} \xi}{\left(1-\alpha_{i}\right) \sigma}\right)^{2}} \frac{\theta_{0}-\xi}{\left(1-\alpha_{i}\right)^{2} \sigma}=0, \lim _{\theta_{0} \rightarrow \infty} \Phi\left(\frac{\theta_{0}-\alpha \xi}{(1-\alpha) \sigma}\right)=$ 1 and, due to (56), we also have $\lim _{\theta_{0} \rightarrow \infty} R \frac{\Phi}{1-\Phi}=e^{\gamma B}$. Taken together, these arguments show that marginal utility $\frac{\partial \mathbb{E} U}{\partial \alpha_{i}}\left(y=\theta_{0}-\alpha_{i} \xi\right)$ of $\alpha_{i}$ vanishes as $y \equiv \theta_{0}-\alpha_{i} \xi$ goes to infinity with $\theta_{0}$.

Strong Fundamental In this paragraph, we use the fact that $\lim _{\theta_{0} \rightarrow+\infty} \frac{\partial \mathbb{E} U}{\partial \alpha_{i}}\left(y=\theta_{0}-\alpha_{i} \xi\right)=0$ to show that the ex-ante incentive to acquire information vanishes as $\theta_{0}$ becomes arbitrarily strong. To this end, we assume that the bond market opens only if an equilibrium exists. That is, we define a critical interest rate $R_{c}$ and the market for bonds only opens if $R \leq R_{c}$. It follows from the bond market equilibrium condition (56) that $R_{c}$ defines a critical signal $y^{*}\left(R_{c}\right)=\theta_{0}-\alpha \xi^{*}$ and a critical signal error $\xi^{*}\left(\theta_{0}\right)$ such that the bond market opens only if $y \geq y^{*}$ or $\xi \leq \xi^{*}$, respectively. If the bond market does not clear, there is no trade and utility is given by $U=U\left(b_{i}=0\right)$. Under these assumptions, ex-ante expected utility is:

$$
V=\int_{-\infty}^{\xi^{*}}\left(-\Phi\left(\frac{\theta_{0}-\alpha_{i} \xi}{\left(1-\alpha_{i}\right) \sigma}\right) e^{-\gamma R b_{i}}-\left(1-\Phi\left(\frac{\theta_{0}-\alpha_{i} \xi}{\left(1-\alpha_{i}\right) \sigma}\right)\right) e^{\gamma b_{i}}\right) \times e^{-\frac{\xi^{2}}{2}} d \xi+\int_{\xi^{*}}^{\infty} U\left(b_{i}=0\right) \times e^{-\frac{\xi^{2}}{2}} d \xi
$$

Recalling the derivative (53), we have marginal expected utility of an increase in signal precision:

$$
\begin{aligned}
\frac{\partial V}{\partial \alpha_{i}}= & \int_{-\infty}^{\xi^{*}} \frac{\partial \mathbb{E} U}{\partial \alpha_{i}}\left(y=\theta_{0}-\alpha_{i} \xi\right) \times e^{-\frac{\xi^{2}}{2}} d \xi \\
& ={ }_{\mid \psi<\xi^{*}} \int_{-\infty}^{\psi} \frac{\partial \mathbb{E} U}{\partial \alpha_{i}}\left(y=\theta_{0}-\alpha_{i} \xi\right) \times e^{-\frac{\xi^{2}}{2}} d \xi+\int_{\psi}^{\xi^{*}} \frac{\partial \mathbb{E} U}{\partial \alpha_{i}}\left(y=\theta_{0}-\alpha_{i} \xi\right) \times e^{-\frac{\xi^{2}}{2}} d \xi \\
& \leq \int_{-\infty}^{\psi} \frac{\partial \mathbb{E} U}{\partial \alpha_{i}}\left(y=\theta_{0}-\alpha_{i} \xi\right) \times e^{-\frac{\xi^{2}}{2}} d \xi+\int_{\psi}^{\xi^{*}} \sup _{y \in\left[y^{*}, \infty\right]} \frac{\partial \mathbb{E} U}{\partial \alpha_{i}}(y) \times e^{-\frac{\xi^{2}}{2}} d \xi
\end{aligned}
$$

and hence,

$$
\lim _{\theta_{0} \rightarrow+\infty} \frac{\partial V}{\partial \alpha_{i}} \leq \lim _{\theta_{0} \rightarrow+\infty} \int_{-\infty}^{\psi} \frac{\partial \mathbb{E} U}{\partial \alpha_{i}}\left(y=\theta_{0}-\alpha_{i} \xi\right) \times e^{-\frac{\xi^{2}}{2}} d \xi+\lim _{\theta_{0} \rightarrow+\infty} \int_{\psi}^{+\infty} \sup _{y \in\left[y^{*}, \infty\right]} \frac{\partial \mathbb{E} U}{\partial \alpha_{i}}(y) \times e^{-\frac{\xi^{2}}{2}} d \xi
$$

From the previous section we recall that $\lim _{\theta_{0} \rightarrow+\infty} \frac{\partial \mathbb{E} U}{\partial \alpha_{i}}\left(y=\theta_{0}-\alpha_{i} \xi\right)=0$ to find 38

$$
\lim _{\theta_{0} \rightarrow+\infty} \frac{\partial V}{\partial \alpha_{i}} \leq(1-\Phi(\psi)) \sup _{y \in\left[y^{*}, \infty\right]} \frac{\partial \mathbb{E} U}{\partial \alpha_{i}}(y), \quad \psi<\xi\left(\theta_{0}\right) .
$$

\footnotetext{
${ }^{38}$ Note that the supremum $\tilde{\Psi}$ is well defined since $\frac{\partial \mathbb{E} U}{\partial \alpha_{i}}$ is (i) a continuous function and (ii) we know from the previous paragraph that $\lim _{y \rightarrow \infty} \frac{\partial \mathbb{E} U}{\partial \alpha_{i}}=0$.
} 
Since the restriction on $\psi$ is $\psi<\xi^{*}\left(\theta_{0}\right)$ and $\lim _{\theta_{0} \rightarrow \infty} \xi^{*}=+\infty$; we can choose an arbitrarily large $\psi$ such that (59) vanishes as $\psi \rightarrow \infty$. That is, the upper bound for the expected utility gain from information (59) vanishes for an arbitrarily strong fundamental.

Weak Fundamental In this paragraph, we add that the incentive to acquire information also vanishes when $\theta_{0}$ is arbitrarily weak. We recall derivative (57):

$$
\frac{\partial V}{\partial \alpha_{i}}=\int_{-\infty}^{\xi^{*}} \frac{\partial \mathbb{E} U}{\partial \alpha_{i}}\left(y=\theta_{0}-\alpha_{i} \xi\right) \times e^{-\frac{\xi^{2}}{2}} d \xi \leq \int_{-\infty}^{\xi^{*}} \tilde{\Psi} \times e^{-\frac{\xi^{2}}{2}} d \xi=\tilde{\Psi} \Phi\left(\xi^{*}\right) .
$$

Where $\Psi \equiv \frac{\partial \mathbb{E} U}{\partial \alpha_{i}}\left(y=\theta_{0}-\alpha_{i} \xi\right)$, and, $\tilde{\Psi} \equiv \sup _{y \in\left[y^{*}, \infty\right]} \frac{\partial \mathbb{E} U}{\partial \alpha_{i}}$. Since $y^{*}\left(R_{c}\right)=\theta_{0}-\alpha_{i} \xi^{*}$ we have $\lim _{\theta_{0} \rightarrow-\infty} \xi^{*}=-\infty$ and, thus, $\lim _{\theta_{0} \rightarrow-\infty} \tilde{\Psi} \Phi\left(\xi^{*}\left(\theta_{0}\right)\right)=0$. That is, the upper bound for the incentive to acquire information (60) vanishes. 